\title{
25 Research Soure \\ Knockdown of IL-6 Plays a Crucial Role in \\ Protection of Hypoxia-Ischemia in Neonatal Rats via Inhibiting Casp3 and BAX Signaling Pathway
}

\section{Ting-Hua Wang}

Sichuan University West China Hospital https://orcid.org/0000-0002-2421-2812

\section{Xue Bai}

Affiliated Traditional Chinese Medical Hospital of Southwest Medical University

\section{Liu-Lin Xiong}

Sichuan University West China Hospital

\section{Ting-Ting Li}

Affiliated Traditional Chinese Medical Hospital of Southwest Medical University

\section{Chang-Le Fang}

Affiliated Traditional Chinese Medical Hospital of Southwest Medical University

\section{Qin-Jiu Xia}

Sichuan University West China Hospital

\section{Qiu-Lin Wang}

Affiliated Traditional Medical Hospital of South Medical University

Si-Jin Yang ( $\square$ ysjimn@sina.com )

Affiliated Traditional Medical Hospital of Southwest Medical University

\section{Research Article}

Keywords: Hypoxia ischemia, interleukin-6, Neonatal, Casp3, BAX

Posted Date: August 26th, 2021

DOI: https://doi.org/10.21203/rs.3.rs-814664/v1

License: (1) (1) This work is licensed under a Creative Commons Attribution 4.0 International License. Read Full License 


\section{Abstract}

Background: The effect of interleukin-6 (IL-6) knockdown on hypoxia-ischemia (HI) of neonatal rat models was investigated to explore the underlying molecular regulation mechanism.

Methods: To establish the HI model, we treated 7days postnatal Sprague-Dawley (SD) rats with the right carotid artery ligation and had them exposed to the environment of $8 \%$ oxygen and $92 \%$ nitrogen for $2 \mathrm{~h}$, respectively. Then, the neurologic function and morphology changes were assessed. Subsequently, IL-6 siRNA lentivirus was injected into cerebral cortex motor area 2 days before $\mathrm{Hl}$; meanwhile, the interference efficiency was detected by quantitative real-time polymerase chain reaction (QRT-PCR) and Western blot. Immunofluorescence staining of Glial fibrillary acidic protein (GFAP), Hexaribonucleotide Binding Protein3 (NeuN) and IL-6 were used to identify the location and interference effect of IL-6. In order to further research the underlying mechanisms, the expressions of downstream molecular including Bcl-2associated X protein (BAX) and Casp3 were examined following IL-6 up-regulation by QRT-PCR.

Results: It was found that both the growth of cortical neurons and the length of axon were promoted after IL-6 interference, and the cell apoptosis was decreased. In addition, the expression of BAX and Casp3 were closely associated with IL-6.

Conclusions: The present findings confirmed that the decreased IL-6 improves the deficiencies in neurologic function and morphology induced by $\mathrm{HI}$, and the potential mechanism may be closely related with the regulation of Casp3 and BAX.

\section{Highlights}

1. The growth of cortical neurons and the length of axon were promoted after IL-6 interference.

2. The cell apoptosis was decreased after IL-6 interference.

3. The expression of BAX and Casp3 were closely associated with IL-6.

4. The decreased IL-6 improves the deficiencies in neurologic function.

\section{Introduction}

Hypoxic-ischemic encephalopathy (HIE) [1] is a clinical symptom in caused by neonate asphyxia, affects 1-5/1000 newborns worldwide. Moreover, HIE is related to cerebral palsy, epilepsy, and cognitive disabilities [2-5]. There are at least two types of injuries that can lead to HI brain injury [6]. Hypoxia and ischemia were the main injury, which interrupted the energy supply of cells and caused cell injury. Hypoxia-ischemia and reoxygenation/reperfusion produced a wide range of "toxic chemicals", such as free oxygen species and inflammatory cytokines, which could lead to additional cell injury [7-9]. Some studies have showed that rats exposed to longer hypoxic periods at post neonatal day3 (PND3) have encephalic and sensorimotor impairments that mimic those observed in preterm infants [10]. There is no effective treatment to minimize $\mathrm{HI}$-induced brain dysfunction in clinic at present. The treatment strategies 
are limited because of the lack of knowledge about the related neuronal networks and the potential mechanisms of $\mathrm{HI}$-induced neurodegeneration.

Interleukin-6 (IL-6) is a kind of cytokine, which embodies its function as a pro-inflammatory cytokine and an anti-inflammatory factor [11]. Furthermore, IL-6 is usually associated with inflammation and pathology, and involved in neurogenesis, and in the response of mature neurons in normal conditions. Moreover, the expression of IL- 6 was showed in several of the major brain diseases and animal models, which indicated that IL-6 could play a role in the observed neuropathology. Therefore, IL- 6 was a clear target of strategic therapies [12]. According to the cellular environment, IL-6 may play a variety of diverse and competing effects including anti-apoptotic, pro-proliferative, growth-inhibitory and inducing differentiation [13]. The IL- 6 binding subunit and IL-6 receptor existed on a membrane-bound in a soluble form, interacted with glycoprotein 130 (gp130) after binding to IL-6 [14]. IL-6-bound gp130 dimerization resulted in the activation of multiple intracellular signal transduction pathways including Janus kinase/signal transducer and activator of transcription (JAK/STAT) $[15,16]$. Consequently, IL-6 may be an early biomarker of severe injury and contribute to the long-term prognostication in infants with HIE [17].

However, the molecular signaling mechanism of IL-6's neuroprotection role in $\mathrm{HI}$ model of neonatal rats is largely unknown. At this study, we explored the mechanism of IL-6 reducing HIE damage and the longterm effects on the neurological function by decreasing apoptosis and improving axonal damage in the development brain and lung after $\mathrm{HI}$ [18]. Our results will provide a novel therapeutic target for $\mathrm{HI}$ gene and molecular treatment.

\section{Materials And Methods \\ 2.1 Animal care}

Timed pregnant female Sprague-Dawley (SD) rats were bought from Animal Centre of Kunming Medical University and placed in individual cages. Postnatal pups were put in their cage with food and water available as libitum throughout the study in a $12 \mathrm{~h}$ light/dark cycle. Then the postnatal day 7 SD rat pups (weighing 10-15g) were employed in this study. All procedures for the use of animal were approved by University Committee on Animal Use and Care. Rat pups were randomly divided into four groups, as shown in Table. The animal study was legally approved by the Animal Care \& Welfare Committee of Kunming Medical University.

\section{Table Animal grouping}




\begin{tabular}{|llll|}
\hline Animal grouping & H112 h & HI24 h \\
\hline Sham group (Sham) & $n=6$ & $n=6$ \\
\hline Hypoxia ischemia group (HI) & $n=6$ & $n=6$ \\
\hline Hypoxia ischemia with no-targeting lentivirus (Negative Control) & $n=6$ & $n=6$ \\
Hypoxia ischemia with IL-6-targeting lentivirus (IL-6-RNAi-LV) & $n=6$ & $n=6$ \\
\hline 2.2 Establishment of HI model & &
\end{tabular}

Before experiments, the neonatal rats were performed to the anesthetized with $3 \%$ isoflurane. After that, the right common carotid artery (CCA) was exposed with a $0.5 \mathrm{~cm}$ skin incision in the midline of the neck and permanently fused by Monopolar Microsurgery Electrocoagulato (Spring Medical Beauty Equipment Co., LTD, Wuhan, China). The rats were returned to their mother to recovery for $1 \mathrm{~h}$ after surgery. Subsequently, the pups were taken into a hypoxic chamber $\left(8 \% \mathrm{O}_{2}, 92 \% \mathrm{~N}_{2}\right)$ for 2 hours (h) to produce $\mathrm{HI}$ injury, then returned to their maternal rats. While in the sham group, the rats were anesthetized with $3 \%$ isoflurane and their CCA was exposed without hypoxia and ischemia [19].

\subsection{Behavioral Analyses}

The neurologic deficits of rats were recorded according to the standard of Longa scores. The higher the score, the more serious the neurological deficit. Briefly, the neurobehavioral scores of the sham group were evaluated at $0,2,4,6,12,24,48 \mathrm{~h}$ after hypoxia-ischemia treatment [20]. The scoring criteria: 1) 0 points: the behavior is completely normal, no symptoms of neurological deficit; 2) 1 point: The bending of the left forelimb could not be fully extended and the neurological function was mild; 3) 2 points: cannot go straight and walk forward when the body continues to turn to the side, moderate neurological deficit; 4) 3 points: Rats inability to stand, fell to the left when standing, severe neurological deficit; 5) 4 points: unable to self-issued, loss of consciousness.

\subsection{Brain Water Content}

At the indicated time points after $\mathrm{HI}$, the brains of rat pups were removed after deep anesthesia and separated into ipsilateral, contralateral and cerebellum portions to measure the water content. Afterwards, wet weight was weighed on electronic balance at once after the brain was removed, then the dry weight was obtained after the tissues dried in an oven at $105^{\circ} \mathrm{C}$ for $24 \mathrm{~h}$ to $36 \mathrm{~h}$ to reach a constant weight (the last two times weighing $<0.2 \mathrm{mg}$ ). Finally, the brain water content was calculated by Elliot formula: the percentage of brain water content $=($ wet weight - dry weight $) /$ wet weight $\times 100 \%$.

\subsection{Tissue harvest}

The tissues of $\mathrm{HI}$ groups were taken at $6 \mathrm{~h}, 12 \mathrm{~h}$ and $24 \mathrm{~h}$ after surgery, while the sham group were obtained at $6 \mathrm{~h}$ post-surgery for Hematoxylin and Eosin Staining (HE staining) and immunohistochemistry. To be specific, the sterile saline was infused from the left ventricular to right auricle until liquid was cleared, then $20 \mathrm{ml}$ of $4 \%$ paraformaldehyde ( $\mathrm{pH} 7.4,0.01 \mathrm{~mol} / \mathrm{L}$ PBS) was 
infused again. Subsequently, the brain was obtained immediately after perfusion and stored in $4 \%$ paraformaldehyde solution for a fixation more than $72 \mathrm{~h}$. The brain and lung were fetched for quantitative real-time polymerase chain reaction QRT-PCR) and western blot (WB). Then the membrane of brain was peeled under a dissecting microscope and the whole hippocampus was quickly taken out on the ice. Finally, the tissues can be stored at $-80{ }^{\circ} \mathrm{C}$ for following experiments.

\subsection{Triphenyl tetrazolium chloride (TTC) Staining}

TTC staining was performed to detect the infract volume of the brain. After the rats were deeply anesthetized and sacrificed after $\mathrm{HI}$ for at least $6 \mathrm{~h}$, the brains were promptly removed. Afterwards, the tissues were frozen at $-20^{\circ} \mathrm{C}$ for $15 \mathrm{~min}$ and sliced into coronal sections of $1-3 \mathrm{~mm}$ thick using a rat brain matrix (Seino Co., Ltd. Beijing, China). Immediately, the above sections were put into $2 \%$ triphenyl tetrazolium chlorides (Sigma Co., St Louis, MO, USA) in the dark and incubated for 10 to 15 min at $37{ }^{\circ} \mathrm{C}$. Then the stained brain slices were taken out and fixed in phosphate buffered solution by $4 \%$ formaldehyde. Finally, Image J software (National Institutes of Health, USA) was used to trace and measure the infarction area of every slice. In addition, the following formula: corrected percentage of infarct volume $=($ contralateral hemispheric volume-ipsilateral non-infarcted volume $) /$ contralateral hemispheric volume, was used to calculate infarct volume [21].

\subsection{Hematoxylin and Eosin (HE) Staining}

For HE staining, the obtained brain tissues were fixed with $4 \%$ paraformaldehyde for more than $72 \mathrm{~h}$ and chopped into $0.5 \mathrm{~cm} \times 0.5 \mathrm{~cm} \times 1 \mathrm{~cm}$ pieces. Then, samples were dehydrated until transparent, immersed in wax, and embedded in paraffın. The paraffin-embedded tissues were cut into $3 \mu \mathrm{m}$ slices using a Rotary Microtome YD-1508R (Jinhua YIDI Medical Appliance Co., Ltd, China). Then, the slices were routinely stained with HE. Next, the stained sections were observed under a light microscope (CX40, Shunyu, Ningbo, China) to detect the pathological changes.

\subsection{Immunohistochemistry}

For immunohistochemical assay, the paraffin embedded sections including hippocampus, cortex and lung were routinely de-paraffinized and rehydrated. Sections were washed four times in PBS and incubated with $10 \%$ goat serum for $1 \mathrm{~h}$ at room temperature to block non-specific binding. Subsequently, sections were incubated with primary antibodies (Abcam, M, ab9324) overnight at $4{ }^{\circ} \mathrm{C}$. Next, the sections were incubated in biotin-conjugated secondary antibody (Goat anti Rabbit lgG, HRP/lgG) for 30 min at $37{ }^{\circ} \mathrm{C}$, then incubated with horseradish peroxidase conjugated streptavidin avidin for $20 \mathrm{~min}$ at 37 ${ }^{\circ} \mathrm{C}$. After that, sections were washed with PBS three times for $5 \mathrm{~min}$ each, and continually incubate with diaminobenzidine (DAB), followed by dehydration, transparentization and mounting. Counterstaining of sections by hematoxylin was also performed. While in the sham group, PBS was used instead of primary antibody. Images were taken with a laser scanning confocal microscope (Nikon, Tokyo, Japan).

Quantification of the immune labeled brain sections was performed separately. For each slice, $\times 200$ magnification photomicrographs were taken to measure the density of IL- 6 . The mean density was 
presented as IOD over the area of interest using Image-Pro plus 6.0 software (Media Cybernetics, Silver Spring, MD, USA), which was described previously.

\subsection{Primary culture of rat cortical neurons and oxygen- glucose deprivation (OGD) model}

Primary cortical neurons were obtained from one-day-postnatal SD rats. Briefly, the cortexes of rats were harvested and cut into approximately $1 \mathrm{~mm}^{3}$ small pieces, then digested with $0.25 \%$ trypsin (Gibco) at 37 ${ }^{\circ} \mathrm{C}$ for 10 min and eluted with $10 \%$ fetal bovine serum (BSA, Gibco). The tissue suspension was centrifuged at $1000 \mathrm{rpm}$ for $10 \mathrm{~min}$. Subsequently, the tissue suspension was centrifuged at $1500 \mathrm{rpm}$ for $5 \mathrm{~min}$, and the pellet in the bottom was resuspended at the bottom using a complete medium (Hyclone) containing DMEM / HIGH GLUCOSE, 10\% fetal bovine serum and 1\% penicillin-streptomycin solution. Next, neurons were seeded at a density of $5 \times 10^{5} \mathrm{cells} / \mathrm{ml}$ in 6 well plates (Corning, USA) coated with polyd-lysine and laminin (Sigma-Aldrich, St. Louis, MO), then incubated under the condition of $37{ }^{\circ} \mathrm{C}$ and $5 \%$ $\mathrm{CO}_{2}$. Thecomplete medium was replaced with $2 \%$ B27 (Invitrogen, $\mathrm{CA}$ ) at $4 \mathrm{~h}$ after incubation. The medium was changed in the next day and then one-half change was made every 3 days.

\subsection{Construction of IL-6 siRNA Lentivirus}

Not-targeting and targeting siRNA were obtained from GeneCopoeia Company (GuangZhou, China). Three 19-nucleotide sequences were designed corresponding to the IL-6 reference sequence (NCBI, NM_031512.2) to specific silencing of IL-6. Non-targeting siRNA was constructed using a 19-nucleotide sequence which is no homology to any mammalian gene sequence as negative control. IL-6 interference lentivirus (MSH028938-HIVU6) was purchased from GeneCopoeia Company (GuangZhou, China) to construct IL-6 siRNA expression vector. In brief, IL- 6 siRNA lentivirus was produced by co-transfected IL-6 expression vector and viral packaging system (gag, pol and env) into 293 T cells according to the manufacturer's protocol. Forty-eight hours after transfection, virus medium was harvested, filtered through a $0.45 \mu \mathrm{m}$ cellulose acetate filter, purified and concentrated by concentration solution (GeneCopoeiaCompany). Finally, the IL-6 siRNA lentivirus was frozen at $-80{ }^{\circ} \mathrm{C}$ for following experiment. Then, IL- 6 siRNA lentivirus ( $5 \mu \mathrm{l}$ for each position) was injected into right side of the lateral ventricle hemisphere, and the injection coordinates were vertical depth $4 \mathrm{~mm}$, right $1.0 \mathrm{~mm}$ relative to bregma and $1.5 \mathrm{~mm}$ from the lambdoid suture. Additionally, the rate of injection was $0.2 \mu \mathrm{l} / \mathrm{min}$.

\subsection{Screen for effective fragment of IL-6 siRNA}

At first, the gene database of IL- 6 from NCBI was gathered. Four silencing RNAs (siRNAs) specifically inhibiting IL-6 gene expression and one nonsense siRNA as negative control (NC) were designed, which were purchased from Ribobio Company (Guangzhou, China). The target messenger RNA (mRNA) sequence of fragment 1 is CCAAGTCCGTC TTCTACAT; fragment 2: CAGGTGCACTTTACGAG TA; fragment 3: CAGCATGAATCCAGCTCGA. In brief, when the PC12 cells were $40 \%$ confluence, three candidate target fragments of IL- 6 siRNA were added into cells. There were four groups in this experiment 
as follows: the normal group, reagent group, negative control group and IL-6 siRNA group. Transfection was performed using SuperFectin ${ }^{\mathrm{TM}}$ II in vitro transfection reagent (Pufei Biotech, China). Briefly, a mix of transfection stock Buffer and siRNA was prepared and $3 \mu$ of SuperFectin ${ }^{\text {TM }} \|$ reagent was added to the mixture. Mixture of IL-6 siRNA (100 nM) was added drop-wise to the appropriate wells, respectively. Another $1.2 \mathrm{ml}$ of fresh culture medium was added at $37^{\circ} \mathrm{C}$ for $24 \mathrm{~h}$ after incubation. Quantitative realtime polymerase chain reaction (QRT-PCR) was performed to validate the inhibition rate of three fragments. Then the siRNA of the highest inhibition rate was transfected into neurons of $\mathrm{HI}$ to explore the function of IL-6. In addition, the morphologic changes of neurons after IL-6 siRNA treatment was observed and collected by a phase contrast microscopy. Non-targeting siRNA was transfected into neurons as negative control.

\subsection{Immunofluorescence Staining (IF)}

To examine the neurons outgrowth, the location and expression of IL-6 following IL-6-RNAi-LV injection, comparative analysis of immunofluorescence staining was performed in the brain tissue section. Briefly, sections were washed three times ( 5 min for each time) in PBS and incubated with $5 \%$ goat serum for $2 \mathrm{~h}$ at room temperature to block non-specific binding. Subsequently, sections were incubated overnight at 4 ${ }^{\circ} \mathrm{C}$ with the following primary antibodies: rabbit anti-GFAP antibody (1:100; Zhongshan Jinqiao, China) together with mouse anti-IL-6 antibody (1:100; Abcam, Cambridge, MA, USA). Then, the sections were washed 3 times with PBS for 5 min each time and incubated with the appropriate secondary antibodies: CY3 anti-rabbit lgG (red), Alexa Fluor 488 anti-mouse lgG (green) and Alexa Fluor 488 anti-rabbit IgG (green) for $2 \mathrm{~h}$ at $37^{\circ} \mathrm{C}$. The sections were washed 3 times for 5 min each with PBS, and stained with DAPI and mounted. Section image was collected using a confocal microscope (Zeiss LSM).

\subsection{TUNEL staining}

A Terminal-deoxynucleoitidyl Transferase Mediated Nick End Labeling (TUNEL) reaction mixture of enzyme solution and labeling solution was added at a ratio of $1: 9(\mathrm{v} / \mathrm{v})$, and the slices were stored at $4{ }^{\circ} \mathrm{C}$ overnight in the dark. After washing with PBS, the neurons were stained with DAPI for $5 \mathrm{~min}$ at room temperature, and photographs were obtained using fluorescence microscopy (Leica, CM1860, Germany). The nuclei of apoptotic neurons were stained in red by TUNEL and the all nuclei of neurons were stained in blue by DAPI. Apoptosis was quantified by determining the percentage of TUNEL/DAPI using ImagePro Plus 6.0 software.

\subsection{Western Blot (WB)}

WB technique was used to detect the expression of IL- 6 protein in cortex and the expression of $\beta$-actin and IL-6 in vitro. The samples were lysed and treated with RIPA lysis buffer (Beyotime, Jiangsu, China). All the samples were centrifuged at $12000 \times \mathrm{g}$ for $10 \mathrm{~min}$ at $4{ }^{\circ} \mathrm{C}$. The total supernatant protein was 
collected and its concentration was determined by BCA protein assay. Then, samples containing $80 \mu \mathrm{g}$ of protein were separated by SDS-PAGE electrophoresis and electro transferred to PVDF membrane. Next, the membranes were blocked with TBST containing $5 \%$ skimmed milk and then incubated with primary antibodies of IL-6 (1:200,Abcam, ab9324, Mouse) overnight at $4{ }^{\circ} \mathrm{C}, \beta$-actin $(1: 1000$, Abcam, USA) was used as a loading control. After washing three times in TBST for 5 min each, the membrane was incubated for $2 \mathrm{~h}$ at room temperature with a secondary antibody (HRP Goat Anti-Mouse IgG, 1:5000, Abbkine, A21010). Finally, the membrane was placed in a chemiluminescent reagent for $1 \mathrm{~min}$, and then exposed to X-ray film cassette. The immune blot was revealed with an ECL Western blot detection kit (Amersham Pharmacia Biotech, Buckinghamshire, England). Densitometry analysis was performed using Image J software.

\subsection{Quantitative real-time polymerase chain reaction (QRT- PCR)}

QRT-PCR was performed to detect the expression of IL-6 in cortical and cultured neurons in vitro. After tissue homogenate, $1 \mathrm{ml}$ Trizol reagent (Takara Bio Inc., Otsu, Japan) was added and total RNA was extracted according to standard specification. Then, $5 \mathrm{ul}$ of the obtained RNA was reversely transcribed to cDNA with the RevertAid ${ }^{\text {TM }}$ First Strand cDNA Synthesis Kit (Thermo, USA). QRT-PCR was performed to examine the messenger RNA (mRNA) level of IL-6. Afterwards, the QRT-PCR was performed. The primer sequences were listed as follows: IL-6: Forward, 5'-GAGGATACCACTCCCAACAGACC-3'; Reverse, 5'AAGTGCAT CATCGTTGTTCATACA-3'. GADDH: Forward, 5'-TGACTTCAACA GCGACACCCA-3'; Reverse, 5'CACCCTGTTGCTGTAGCCAAA-3'. BAX: Forward, 5'-TGGAGCTGCAGAGGATGATT5-3'; Reverse, 5'-CA GGGCCTTGAGCACCAGTT-3'. Casp3: Forward, 5'-TTCTTCAGAGG CGACTACT-3'; Reverse, 5'TCCCACTGTCTGTCTCAAT-3'. AKT1: Forward, 5'-AGTCCCCACTCAACAACTTCT-3', Reverse, 5'-GAAGG TGCGCTCAATGACTG-3'. STAT3: Forward, 5'-GTGCAGGATCTAG AACA GAA-3'; Reverse, 5'GACTGGTTGTTTCCATTCAG-3'. ERK1: Forward, 5'-CAAGA ACAAGACCAACATGAAT-3'; Reverse, 5'-GGTA GGACACAAACTTGTAG-3'. JAK1: Forward, 5'-TTCACTGGAGTAT CTGTTTG-3'; Reverse, 5'CAACTGCATCTTCTTCATCAT-3'. Subsequently, the reaction was carried out in a DNA thermal cycler (ABI 7300) based on the following standard protocol: a denaturation step of $95^{\circ} \mathrm{C}$ for 3 min; 45 cycles of 95 ${ }^{\circ} \mathrm{C}$ for $15 \mathrm{~s}$ and annealing temperature at $60{ }^{\circ} \mathrm{C}$ for $30 \mathrm{~s}$. The threshold cycle of each sample was recorded, and data were analyzed by normalization to GAPDH values using the $2-\triangle \triangle \mathrm{Ct}$ method.

\subsection{Statistical analysis}

All data in the experiment were presented as mean \pm SD and were analyzed using SPSS 20.0. Comparisons between two groups were analyzed by Independent-Samples T-test. Non-parametric comparisons between three or more groups were made with one-way ANOVA test. $P<0.05$ was considered statistically significant. 


\section{Results}

\subsection{HI could cause brain injury.}

Brain injury (including Brain swelling, brain water content, cerebral infarction) was detected after operation (Fig. 1A). TTC staining in the HI group showed a clear cerebral infarction on the right side of the brain compared with the sham group (Fig. 1B). It was found that brain swelling markedly increased post $\mathrm{HI}$, and there was significant difference at $24 \mathrm{~h}$ in the $\mathrm{HI}$ group compared with the sham group. In addition, brain swelling at $24 \mathrm{~h}$ was more severe than that of $12 \mathrm{~h}$ in the HI group $(p<0.05$, Fig. $1 \mathrm{C})$. Infarct percentage indicated that the area of cerebral infarction significantly increased at both 12 and 24 h post $\mathrm{HI}(\mathrm{p}<0.05$, Fig. 1C). Brain water content increased in the HI-ipsilateral group when compared with the sham group ( $p<0.05$, Fig. 1D), however, there was no obvious difference in the HI-contralateral and HI-cerebellum groups, when compared with the sham group ( $p>0.05$, Fig. 1D). In addition, the zea-longa scores were performed after $\mathrm{HI}$ operation in rats. Compared with the sham group, the zea-longa score of $\mathrm{HI}$ rats was increased after $0-4 \mathrm{~h}$ postoperative monitoring. The zea-longa score was decreased after 24 $\mathrm{h}$ in the $\mathrm{HI}$ group, but still higher than in the sham group (Fig. 1E).

\section{2 $\mathrm{HI}$ could cause severe brain edema and neurological deficit aggravation.}

In order to determine the degree of brain edema caused by HI, HE staining was employed to assess the morphology changes of the brain tissue. Compared with the sham group, brain swelling was markedly more severe in the HI group (Fig. 2A). In addition, HE staining showed swelling or necrosis and illegible borders of cell in the HI group (Fig. 2B). To explore the cause of neurological deficit aggravation in $\mathrm{HI}$ rats, we also detected apoptosis by TUNEL staining. It was manifested that the number of TUNEL-positive cells markedly increased in the HI group compared with sham one (Fig. 2C).

\subsection{The number of IL-6 increased cortex and hippocampus after $\mathrm{HI}$}

Immunohistochemistry was performed to semi-quantify the level of IL-6 in the hippocampus and cortex in the sham and $\mathrm{HI}$ groups. Results demonstrated that the number of IL-6-positive cells in the hippocampus and cortex significantly increased in the HI group compared with those of the sham group (Fig. 3A). Meanwhile, there was significant difference in IL-6-positive cell number between the hippocampus-left and cortex-right sub-group ( $p<0.05$, Fig. 3B).

\subsection{The expression of IL-6 mRNA and protein increased after $\mathrm{HI}$ in cortex-right and hippocampus-right.}

To detect the crucial genes associated with HI, IL-6 mRNA in the hippocampus and cortex were examined by QRT-PCR and the results indicated that the mRNA relative expression of IL-6 was evidently increased following $\mathrm{HI}$ in the hippocampus and cortex compared with that of sham group respectively (Fig. 4A, B, C, 
and D). Alternatively, the level of IL- 6 mRNA increased at $6 \mathrm{~h}, 12 \mathrm{~h}$ and $24 \mathrm{~h}$ post $\mathrm{HI}$ in the cortex-right subgroup ( $p<0.05$, Fig. $4 A$ ), as well as $12 \mathrm{~h}$ and $24 \mathrm{~h}$ post $\mathrm{HI}$ in the hippocampus-right sub-group $(\mathrm{p}<0.05$, Fig. 4B) when compared with corresponding time-spot sub-group in the sham group respectively.

Subsequently, WB was performed to identify the changes of IL- 6 protein expression. It was found that the IL-6 protein level was distinctly increased following $\mathrm{HI}$, which was consistent with gene expression (Fig. $4 \mathrm{E}, \mathrm{F}$ ). Quantitative analysis showed that the relative OD value of IL-6 after $\mathrm{HI}$ in the cortex-right and hippocampus-right sub-groups was notably increased compared with the sham group, respectively $(p<$ 0.05, Fig. 4F).

\subsection{The location of IL-6 was consisting in neuron and GFAP.}

Immunofluorescence was performed to confirm the location of IL-6 in the hippocampus and cortex in the sham group and HI group. The number of IL-6 positive cells, the neuron and GFAP greatly increased in the HI group compared with those in the sham group (Fig. 5).

\subsection{The relative expressions of BAX and Casp3 were associated with IL-6 siRNA.}

QRT-PCR showed the relative expression of BAX decreased in cortex-right but increased in hippocampusright in the comparison of negative-control and IL- 6 siRNA groups, moreover, the differences were statistically significant $(p<0.05$, Fig. $6 A, B)$. In addition, the relative expression of Casp3 increased in cortex-right $(p<0.05)$ in the comparison of negative-control and IL-6 siRNA groups, however there was no statistical difference in the expression change of hippocampus (Fig. 6C, D). In addition, it was found that, both the relative expression of BAX and Casp3 in lung decreased after IL- 6 siRNA $(p<0.05$, Fig. $6 E, F)$. Double immunofluorescence labeling demonstrated that the co-localization for IL-6 (green) and Casp3 (Red) (Fig. 6G).

\subsection{F2 was the effective interference on IL-6 gene}

In order to determine the effective interference fragment on IL- 6 gene, we transfected F1, F2 and F3 interference fragment into PC12 cells respectively (Fig. 7A). Then RT-PCR was utilized to calculate the interference efficiency, and we found that F2 interference fragment was most efficient $(p<0.05$, Fig. 7C). Moreover, the expression of IL- 6 was up-regulated after OGD treatment $(p<0.05$, Fig. 7B). Afterwards, to verify whether the F2 interference fragment could specially inhibit IL-6 gene expression, F2 fragment was transfected into neurons once again (Fig. 7D). QRT-PCR was employed to assess the interference efficiency. Notably, we found that the expression of IL-6 was significantly down-regulated by F2 interference fragment $(p<0.05$, Fig. 7E).

\subsection{The growth of cortical neurons was promoted after interference with IL-6 under OGD conditions.}

In order to explore the effect of OGD on cortical neurons, we primarily cultured rat cerebral cortex cells. Cells were homogenously distributed within or along the walls, some cells emerged as spherical buds, 
some as laminar aggregates, and others were dispersed as single cells over time (Fig. 8A). To investigate whether IL-6 siRNA could promote the growth and induce the apoptosis of neurons, immunofluorescence staining for TUJ1 and TUNEL were conducted respectively. The fluorescent graphs revealed that the level of TUJ1 was significantly reduced in the OGD + NC groups (Fig. 8B), while apoptotic cells were remarkably decreased after IL-6 siRNA treatment (Fig. 8C), when compared with OGD + NC conditions. Image-analysis showed that the number of neurons and the length of axons substantially increased after interference with IL- 6 under OGD conditions compared with the OGD + NC group $(p<0.05$, Fig. 8D, E). Nevertheless, the apoptosis of neurons became markedly less than the OGD + NC group after interference with IL-6 under OGD conditions ( $p<0.05$, Fig. 8F). These results showed that down-regulation of IL-6 improved the deficiencies of neurologic function and morphology induced by $\mathrm{HI}$.

\subsection{BAX and Casp3 were associated with IL-6.}

We applied an interaction network analysis for IL-6 gene to predict their interactions and associations based on protein expression using Genemania software (Fig. 9A). To get a better understanding of the association among downregulation of IL- 6 and BAX, Casp3, we performed quantitative analysis of QRTPCR and found that the levels of BAX and Casp3 were prominently down-regulated in the OGD + IL-6siRNA group compared with the OGD + NC group under OGD conditions $(p<0.05$, Fig. 9B, C).

\subsection{The expression of AKT, STAT3, ERK1, and JAK1 increased after interference of IL-6.}

IL-6 gene is involved in different biological processes. To ascertain the effect of IL-6 gene and OGD on various downstream genes, we generated Si-RNA-silenced IL- 6 gene by genetic engineering technology. IL6 siRNA was synthesized and transfected into neurons in vitro, and then QRT-PCR was employed to determine the mRNA expression of different cytokines. Interestingly, we found that AKT, STAT3, ERK1 and JAK1 were significantly increased after IL-6 siRNA compared with negative control group $(p<0.05$, Fig. 10A, B, C, D).

\section{Discussions}

This study demonstrated that $\mathrm{HI}$ led to neurologic deficit, brain swelling and infarction, which accompanied up-regulated IL-6. However, this pathological phenomenon was reversed and attenuated after down-regulated IL- 6 by IL- 6 siRNA lentivirus in vivo and IL-6-RNAi-LV lentivirus in vitro. In addition, our findings suggested that Casp3 and BAX may be the down-stream signaling molecule of the IL- 6 and associated with regulation of $\mathrm{HI}$.

\subsection{Successful establishment of HI animal model}

In this study, the primary culture was administrated free-serum medium and exposed to $2 \% \mathrm{O}_{2}$ and $98 \%$ $\mathrm{N}_{2}$ for $12 \mathrm{~h}$ and $24 \mathrm{~h}$ to establish cell model of OGD. Seven days postnatal rats were subjected to the right carotid artery ligation and subsequently exposed to $8 \% \mathrm{O}_{2}$ and $92 \% \mathrm{~N}_{2}$ for $12 \mathrm{~h}$ and $24 \mathrm{~h}$, respectively, to 
establish animal model of HI. We examined the variation of morphological and functional of neuron and brain tissue following $\mathrm{HI}$, and cellular swelling, inflammatory cell infiltration and cellular necrosis were observed, and margin of cells was unclear in the HI group. Moreover, the degree of neural dysfunction was present in time-dependent manner under $\mathrm{HI}$ intervention; the water quantity of brain tissue also gradually increased with the extent of time of $\mathrm{HI}$. Given that those changes had a statistical significance when compared with their control group, this indicated that $\mathrm{HI}$ model was successfully established. Neonatal $\mathrm{HI}$ not only induces periventricular white matter injury known as periventricular leukomalacia (PVL) and significant neuronal death [22, 23], but also stimulates cerebral microvascular responses and BBB damage [24-26], ultimately leading to neurological functional deficits [27].

\subsection{Behavioral Analyses}

The $\mathrm{HI}$ group had an average score of 2.5 , showing postural postulation and turning to the injured side. These results suggest brain injury and deterioration of neurological function in rats after $\mathrm{HI}$.

\section{IL-6 may play a neuroprotective role by down-regulating its expression in brain tissues}

In this study, we found that the mRNA expression level of IL-6 was markedly increased following $\mathrm{HI}$ in the hippocampus and cortex compared with that of the sham group respectively, which was consistent with the augmentation of IL- 6 positive neurons in the hippocampus and cortex of $\mathrm{HI}$ rats. Increased expression of IL- 6 is evident, particularly in the lung of animals subjected to hypoxic preconditioning. To explore the role of IL-6 in HI encephalopathy, we designed siRNA and constructed the lentevirus vector of IL- 6 siRNA to interfere its expression. After microinjection the lentivirus in the cortex motor area of $\mathrm{HI}$ rat, western blot and immunofluorescent staining results showed IL-6 protein level was down-regulated significantly in IL6-RNAi-LV group when compared with that in the control group. Moreover, the brain edema, swelling neuron and neurologic deficit following $\mathrm{HI}$ were attenuated with down-regulated IL-6. It is therefore clear that down-regulated IL- 6 plays a crucial and active role in the restoration of brain lesion and lung tissue in rat $\mathrm{HI}$ condition. It is concluded from this study that IL-6 may play its neuroprotective role by downregulating its expression in brain tissues, which contrary to the results of our previous study [16], in which IL-1 $\beta$ down regulation improves hypoxia-ischemia brain associated with IL- 6 up-regulation in cell and rat models. This may be due to the different target tissue examined and time point. Additional, recent studies have found that cortical neurons upregulated IL-6 mRNA and protein, and IL-6R after pMCAO [28]. However, some researchers [29] suggested that breviscapine is effective in promoting neurological behavior after traumatic brain injury and the underlying molecular mechanism may be associated with the suppression of interleukin-6. Further study was performed to explore the molecular mechanism of beneficial effects of down-regulation of IL-6 on HI.

\subsection{The signaling molecule associated with IL- 6 may achieves its neuroprotective function after $\mathrm{HI}$ by down- regulating the expression of IL-6}


Simultaneously, QRT-PCR showed that the levels of both Casp3 and BAX were prominently decreased in both cortex and hippocampus after IL-6 siRNA treatment compared with the OGD + NC group. It implies that the presence of IL-6 could naturally promote the expression of Casp3 and BAX. We found that Casp3 and BAX were significantly increased when IL- 6 expression was reduced. In recent studies, hypoxicischemic brain injury is often delayed and involves both apoptotic and immune regulatory mechanisms [30]. Similarly, some studies reported that phycticpreconditioning (PC) seemed to have an acute protective effect on the liver by modulating the inflammatory, apoptotic and anti-oxidative response [31]. Moreover, it was found that IL-6-mediated expression of STC-1 is one molecular mechanism of hypoxic preconditioning-induced tolerance to brain ischemia [32]. Regulating the levels of IL-6, Fas and BDNF in the brain to maintain at reasonable levels may be the mechanism [33]. Some studies have shown that HIBD induces IL-10 secretion from astrocytes to exert a paracrine-induced anti-apoptotic effect on injured neurons via the TLR2/NFKB signaling pathway, which may improve learning and memory dysfunction after ischemic injury [34]. Previous researchers [35] found that the neuroprotective effect of MSC transplantation in neonatal HIBD rats is partly mediated by IL-6 to enhance anti-apoptosis of injured astrocytes via the IL-6/STAT3 signaling pathway. As it is well known that Casp3 and BAX are mainly apoptotic genes, down-regulation of IL-6 may plays its neuroprotective role via prohibiting Casp3 and BAX gene expression.

\section{Conclusion}

These findings suggest that IL- 6 may be a target gene therapy of HI brain edema in neonates. The method of down-regulation of IL- 6 by RNA interference during HI may protect against brain edema, stoke and other brain disorders via up-regulation of Casp3 and BAX expression. Our results will pave a novel way for developing of genetic intervention strategies for the clinical therapy of $\mathrm{HI}$ in sooner future.

\section{Abbreviations}

AKT: Protein kinase $B$

BAX: Bcl-2-associated X protein

CCA囚common carotid artery

DAPI: 4', 6-diamidino-2-phenylindole

ERK1: Mitogen-activated protein kinase 3

F1: treatment with No.1 siRNA fragment

F2: treatment with No.2 siRNA fragment

F3: treatment with No.3 siRNA fragment 
GFAP: Glial fibrillary acidic protei

gp130『glycoprotein 130

HE: hematoxylin and eosin

HI: hypoxia-ischemia

HI-6 h: 6 hour after hypoxia-ischemia

HI-12 h: 12 hours after hypoxia-ischemia

HI-24 h: 24 hours after hypoxia-ischemia

HIEखHypoxic-ischemic encephalopathy

IF囚Immunofluorescence Staining

JAK1: Janus kinase 1

IL-6si: silencing IL-6

Interleukin-6 (IL-6)

NC: negative control.

NeuN: Hexaribonucleotide Binding Protein-3

OGD: oxygen-glucose deprivation

PC『phycticpreconditioning

QRT-PCR: quantitative real-time polymerase chain reaction

siRNA: silencing RNA.

SD\&Sprague-Dawley

STAT3: Signal transducer and activator of transcription 3.

TTC: Triphenyl tetrazolium chloride

Tuj1: Neuronal Class III $\beta$-Tubulin

TUNEL: Terminal-deoxynucleoitidyl Transferase Mediated Nick End Labeling

WB囚western blot 


\section{Declarations}

\section{Funding}

This study was supported by the program Innovative Research Team in Science and Technology in Yunnan province (2017HC007) and National Natural Science Foundation of China (No. NSF 81601074), Sichuan provincial scientific grant (2017SZ0145).

\section{Authors' contributions}

Liu-Lin Xiong, Xue-Rong Zhang, Lu-Lu Xue, Xue Bai and Si-Jin Yang conceived and performed experiments, Liu-Lin Xiong, Hao-Li Zhou, Xue-Rong Zhang, Lu-Lu Xue, Zheng Ma analyzed and interpreted the data. Hao-Li Zhou, Ming-An Yang, Xue Bai and Si-Jin Yang revised and submitted the manuscript. All authors read and approved the final manuscript.

\section{Acknowledgments}

We would like to thank Professor Fei Liu for her fund support and excellent technical guidance.

\section{Declaration of interest}

None

\section{References}

1. E. Rocha-Ferreira, M. Hristova, Antimicrobial peptides and complement in neonatal hypoxia-ischemia induced brain damage, Frontiers in immunology 6 (2015) 56. doi:10.3389/fimmu.2015.00056.

2. J. Armstrong-Wells, T.J. Bernard, R. Boada, M. Manco-Johnson, Neurocognitive outcomes following neonatal encephalopathy, NeuroRehabilitation 26(1) (2010) 27-33. doi:10.3233/nre-2010-0533.

3. K.H. Chang, C.M. Yeh, C.Y. Yeh, C.C. Huang, K.S. Hsu, Neonatal dexamethasone treatment exacerbates hypoxic-ischemic brain injury, Molecular brain 6 (2013) 18. doi:10.1186/1756-6606-6-18.

4. H.C. Glass, K.J. Hong, E.E. Rogers, R.J. Jeremy, S.L. Bonifacio, J.E. Sullivan, A.J. Barkovich, D.M. Ferriero, Risk factors for epilepsy in children with neonatal encephalopathy, Pediatric research 70(5) (2011) 535-40. doi:10.1203/PDR.0b013e31822f24c7.

5. R.C. Vannucci, Hypoxic-ischemic encephalopathy, American journal of perinatology 17(3) (2000) 113-20. doi:10.1055/s-2000-9293.

6. Z. Wang, H. Zhao, S. Peng, Z. Zuo, Intranasal pyrrolidine dithiocarbamate decreases brain inflammatory mediators and provides neuroprotection after brain hypoxia-ischemia in neonatal rats, Experimental neurology 249 (2013) 74-82. doi:10.1016/j.expneurol. 2013. 08.006.

7. C.L. Allen, U. Bayraktutan, Oxidative stress and its role in the pathogenesis of ischaemic stroke, International journal of stroke : official journal of the International Stroke Society 4(6) (2009) 461-70. doi:10. 1111/j.1747-4949.2009.00387.x. 
8. S.E. Lakhan, A. Kirchgessner, M. Hofer, Inflammatory mechanisms in ischemic stroke: therapeutic approaches, Journal of translational medicine 7 (2009) 97. doi:10.1186/1479-5876-7-97.

9. Y. Lampl, M. Boaz, R. Gilad, M. Lorberboym, R. Dabby, A. Rapoport, M. Anca-Hershkowitz, M. Sadeh, Minocycline treatment in acute stroke: an open-label, evaluator-blinded study, Neurology 69(14) (2007) 1404-10. doi:10.1212/01.wnl.0000277487.04281.db.

10. L.E. Duran-Carabali, D.M. Arcego, F.K. Odorcyk, L. Reichert, J.L. Cordeiro, E.F. Sanches, L.D. Freitas, C. Dalmaz, A. Pagnussat, C.A. Netto, Prenatal and Early Postnatal Environmental Enrichment Reduce Acute Cell Death and Prevent Neurodevelopment and Memory Impairments in Rats Submitted to Neonatal Hypoxia Ischemia, Molecular neurobiology 55(5) (2018) 3627-3641. doi:10.1007/s12035017-0604-5.

11. A.C. Ferguson-Smith, Y.F. Chen, M.S. Newman, L.T. May, P.B. Sehgal, F.H. Ruddle, Regional localization of the interferon-beta 2/B-cell stimulatory factor 2/hepatocyte stimulating factor gene to human chromosome 7p15-p21, Genomics 2(3) (1988) 203-8.

12. M. Erta, A. Quintana, J. Hidalgo, Interleukin-6, a major cytokine in the central nervous system, International journal of biological sciences 8(9) (2012) 1254-66. doi:10.7150/ijbs.4679.

13. K. Gertz, G. Kronenberg, R.E. Kalin, T. Baldinger, C. Werner, M. Balkaya, G.D. Eom, J. Hellmann-Regen, J. Krober, K.R. Miller, U. Lindauer, U. Laufs, U. Dirnagl, F.L. Heppner, M. Endres, Essential role of interleukin-6 in post-stroke angiogenesis, Brain: a journal of neurology 135(Pt 6) (2012) 1964-80. doi:10.1093/brain/aws075.

14. E. Juttler, V. Tarabin, M. Schwaninger, Interleukin-6 (IL-6): a possible neuromodulator induced by neuronal activity, The Neuroscientist: a review journal bringing neurobiology, neurology and psychiatry 8(3) (2002) 268-75. doi:10.1177/1073858402008003012.

15. P.C. Heinrich, I. Behrmann, S. Haan, H.M. Hermanns, G. Muller-Newen, F. Schaper, Principles of interleukin (IL)-6-type cytokine signalling and its regulation, The Biochemical journal 374(Pt 1) (2003) 1-20. doi:10.1042/bj20030407.

16. S. Liu, S. Zhu, Y. Zou, T. Wang, X. Fu, Knockdown of IL-1 beta improves hypoxia-ischemia brain associated with IL-6 up-regulation in cell and animal models, Molecular neurobiology 51(2) (2015) 743-52. doi:10.1007/s12035-014-8764-z.

17. C.E. Ahearne, R.Y. Chang, B.H. Walsh, G.B. Boylan, D.M. Murray, Cord Blood IL-16 Is Associated with 3Year Neurodevelopmental Outcomes in Perinatal Asphyxia and Hypoxic-Ischaemic Encephalopathy, Developmental neuroscience 39(1-4) (2017) 59-65. doi:10.1159/000471 508.

18. W. Chen, R. Hartman, R. Ayer, S. Marcantonio, J. Kamper, J. Tang, J.H. Zhang, Matrix metalloproteinases inhibition provides neuroprotection against hypoxia-ischemia in the developing brain, Journal of neurochemistry 111(3) (2009) 726-36. doi:10.1111/j.1471-4159.2009.06362.x.

19. L. Li, Y. Qu, J. Li, Y. Xiong, M. Mao, D. Mu, Relationship between HIF-1alpha expression and neuronal apoptosis in neonatal rats with hypoxia-ischemia brain injury, Brain research 1180 (2007) 133-9. doi:10.1016/j.brainres.2007.08.059. 
20. Y.C. Meng, Z.Y. Ding, H.Q. Wang, L.P. Ning, C. Wang, Effect of microRNA-155 on angiogenesis after cerebral infarction of rats through AT1R/VEGFR2 pathway, Asian Pacific journal of tropical medicine 8(10) (2015) 829-35. doi:10.1016/j.apjtm.2015.09.009.

21. Y. Qu, J. Wu, D. Chen, F. Zhao, J. Liu, C. Yang, D. Wei, D.M. Ferriero, D. Mu, MiR-139-5p inhibits HGTD-P and regulates neuronal apoptosis induced by hypoxia-ischemia in neonatal rats, Neurobiology of disease 63 (2014) 184-93. doi:10.1016/j.nbd.2013.11.023.

22. M.A. Hossain, Hypoxic-ischemic injury in neonatal brain: involvement of a novel neuronal molecule in neuronal cell death and potential target for neuroprotection, International journal of developmental neuroscience : the official journal of the International Society for Developmental Neuroscience 26(1) (2008) 93-101. doi:10. 1016/j.ijdevneu.2007.08.013.

23. M. Zonouzi, J. Scafidi, P. Li, B. McEllin, J. Edwards, J.L. Dupree, L. Harvey, D. Sun, C.A. Hubner, S.G. Cull-Candy, M. Farrant, V. Gallo, GABAergic regulation of cerebellar NG2 cell development is altered in perinatal white matter injury, Nature neuroscience 18(5) (2015) 674-82. doi:10.1038/nn.3990.

24. A.A. Baburamani, C.J. Ek, D.W. Walker, M. Castillo-Melendez, Vulnerability of the developing brain to hypoxic-ischemic damage: contribution of the cerebral vasculature to injury and repair?, Frontiers in physiology 3 (2012) 424. doi:10.3389/fphys.2012.00424.

25. C.J. Ek, B. D'Angelo, A.A. Baburamani, C. Lehner, A.L. Leverin, P.L. Smith, H. Nilsson, P. Svedin, H. Hagberg, C. Mallard, Brain barrier properties and cerebral blood flow in neonatal mice exposed to cerebral hypoxia-ischemia, Journal of cerebral blood flow and metabolism : official journal of the International Society of Cerebral Blood Flow and Metabolism 35(5) (2015) 818-27. doi:10.1038/jcbfm.2014.255.

26. Y.C. Hsu, Y.C. Chang, Y.C. Lin, C.I. Sze, C.C. Huang, C.J. Ho, Cerebral microvascular damage occurs early after hypoxia-ischemia via nNOS activation in the neonatal brain, Journal of cerebral blood flow and metabolism : official journal of the International Society of Cerebral Blood Flow and Metabolism 34(4) (2014) 668-76. doi:10.1038/jcbfm. 2013.244.

27. Q. Ma, C. Dasgupta, Y. Li, L. Huang, L. Zhang, MicroRNA-210 Suppresses Junction Proteins and Disrupts Blood-Brain Barrier Integrity in Neonatal Rat Hypoxic-Ischemic Brain Injury, International journal of molecular sciences 18(7) (2017).doi:10.3390/ijms18071356.

28. M.H. Gronhoj, B.H. Clausen, C.D. Fenger, K.L. Lambertsen, B. Finsen, Beneficial potential of intravenously administered IL- 6 in improving outcome after murine experimental stroke, Brain, behavior, and immunity 65 (2017) 296-311.doi:10.1016/j.bbi.2017.05.019.

29. L. Jiang, Y. Hu, X. He, Q. Lv, T.H. Wang, Q.J. Xia, Breviscapine reduces neuronal injury caused by traumatic brain injury insult: partly associated with suppression of interleukin-6 expression, Neural regeneration research 12(1) (2017) 90-95.doi:10.4103/1673-5374.198990.

30. Y. Carlsson, A.L. Leverin, M. Hedtjarn, X. Wang, C. Mallard, H. Hagberg, Role of mixed lineage kinase inhibition in neonatal hypoxia-ischemia, Developmental neuroscience 31(5) (2009) 420-6. doi:10.1159/000232560. 
31. E. Vlassaks, M. Nikiforou, E. Strackx, M. Hutten, O. Bekers, D. Gazzolo, G. Li Volti, P. MartinezMartinez, B.W. Kramer, A.W. Gavilanes, Acute and chronic immunomodulatory changes in rat liver after fetal and perinatal asphyxia, Journal of developmental origins of health and disease 5(2) (2014) 98-108.doi:10.1017/s2040174413000561.

32. J.A. Westberg, M. Serlachius, P. Lankila, M. Penkowa, J. Hidalgo, L.C. Andersson, Hypoxic preconditioning induces neuroprotective stanniocalcin-1 in brain via IL-6 signaling, Stroke 38(3) (2007) 1025-30. doi:10.1161/01.STR.0000258113.67252.fa.

33. Y. Liu, X. Zhang, Y. Dai, C. Shu, P. Qu, Y.X. Liu, L. Yang, T.Y. Li, [Effects of bone marrow mesenchymal stem cells on learning and memory functional recovery in neonatal rats with hypoxic-ischemic brain damage], Zhonghua er ke za zhi = Chinese journal of pediatrics 46(9) (2008) 648-53.

34. M.L. He, Z.Y. Lv, X. Shi, T. Yang, Y. Zhang, T.Y. Li, J. Chen, Interleukin-10 release from astrocytes suppresses neuronal apoptosis via the TLR2/NFkappaB pathway in a neonatal rat model of hypoxicischemic brain damage, Journal of neurochemistry (2017). doi:10.1111/jnc.14126.

35. Y. Gu, M. He, X. Zhou, J. Liu, N. Hou, T. Bin, Y. Zhang, T. Li, J. Chen, Endogenous IL-6 of mesenchymal stem cell improves behavioral outcome of hypoxic-ischemic brain damage neonatal rats by supressing apoptosis in astrocyte, Scientific reports 6 (2016) 18587. doi:10.1038/ srep18587.

\section{Figures}


$\mathbf{A}$

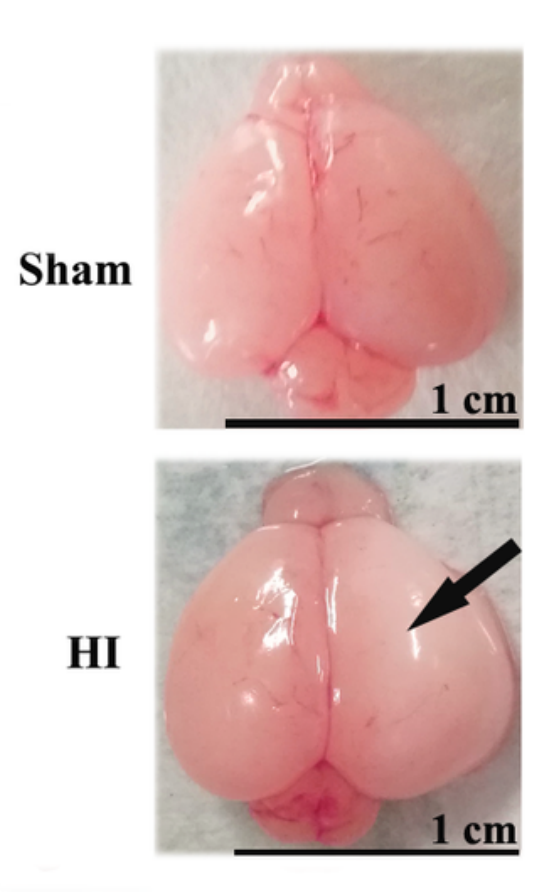

\section{B}
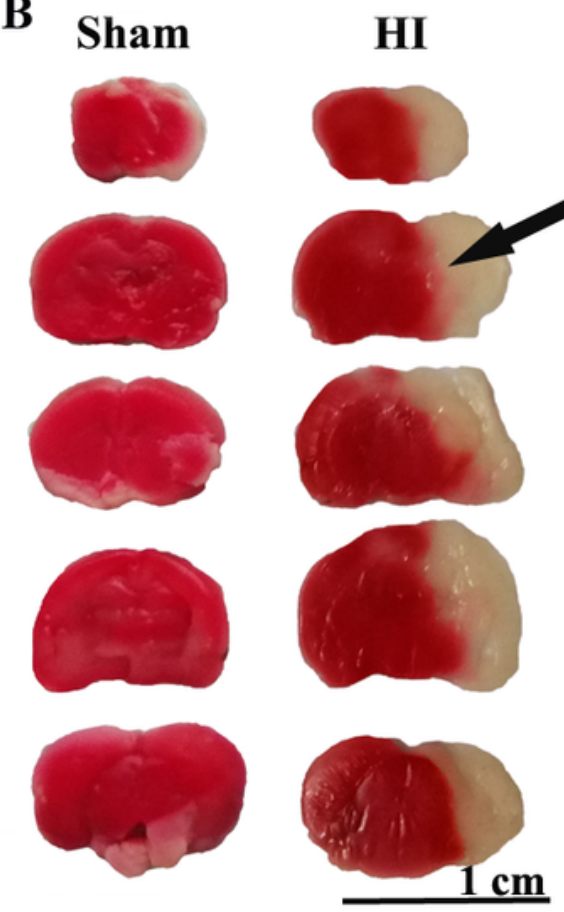

C

D
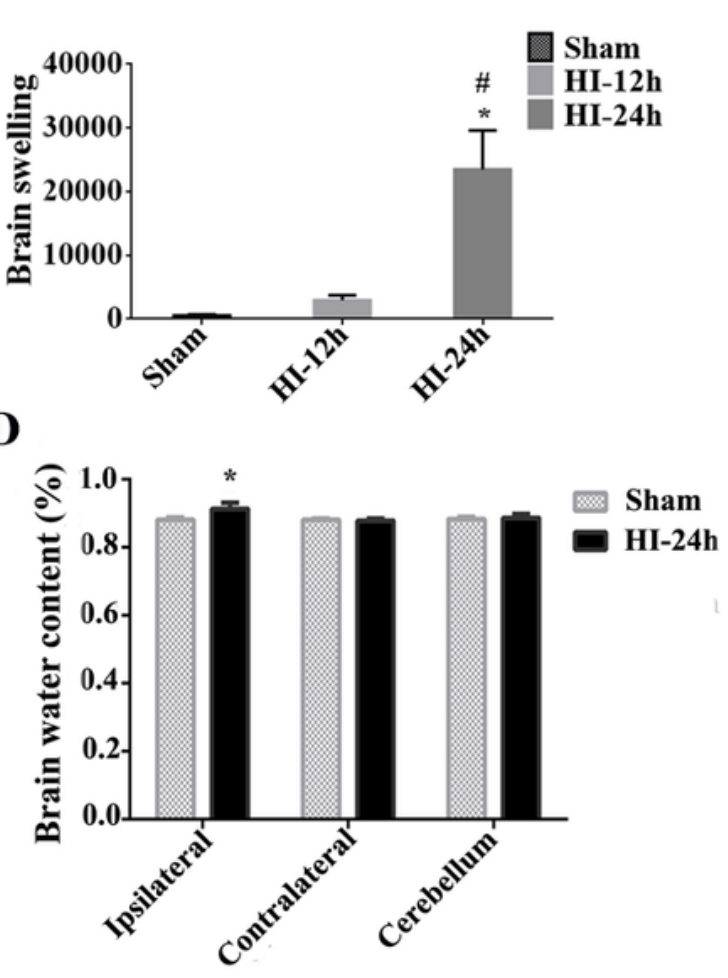

$\mathbf{E}$

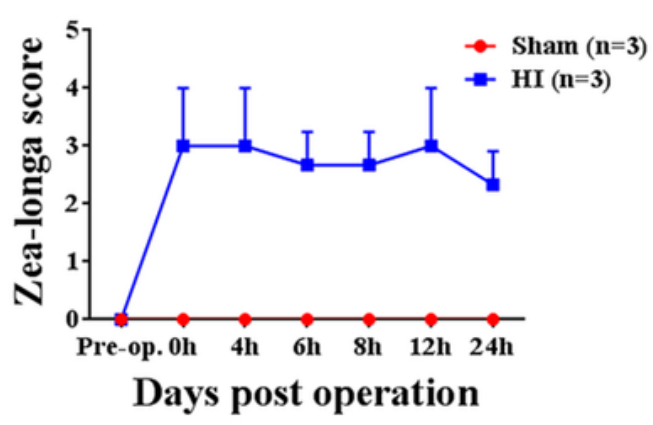

\section{Figure 1}

The injury of brain following $\mathrm{HI}$ surgery $(\mathrm{A})$ The general morphology of brain tissue after $\mathrm{HI}$ surgery. Scale bar $=1 \mathrm{~cm}$. (B) TTC staining in the HI group showed a clear cerebral infarction on the right side of the brain compared with the sham group (Fig 1B). Scale bar $=1 \mathrm{~cm}$. (C) The brain swelling and infarct percentage from the model group revealed remarkable ischaemic injury 12 and $24 \mathrm{~h}$ after HI operation. *p $<0.05$ vs. sham group, \#p < 0.05 vs. HI-12 h group. Scale bar $=1 \mathrm{~cm}$. (D) Brain water content of HI- 
ipsilateral, $\mathrm{HI}$-contralateral and cerebellum standing on the left. (E) Zea-longa scores were performed after $\mathrm{HI}$ operation in rats. Compared with the sham group, the zea-longa score of $\mathrm{HI}$ rats was increased after 0$4 \mathrm{~h}$ postoperative monitoring. The zea-longa score was decreased after $24 \mathrm{~h}$ in the HI group, but still higher than in the sham group (Fig 1E). HI: hypoxia-ischemia. TTC: Triphenyl tetrazolium chloride. MRI: Magnetic resonance imaging. HI-6 h: 6 hour after hypoxia-ischemia, $\mathrm{HI}-12$ h: 12 hours after hypoxiaischemia $\mathbb{H I}-24 \mathrm{~h}$ : 24 hours after hypoxia-ischemia. All data are presented as mean $\pm \mathrm{SD}$. ${ }^{\star} \mathrm{p}<0.05, \# \mathrm{p}<$ 0.05. *: Sham vs. HI-24 h, \#: HI-12 h vs. HI-24 h. $\mathrm{n}=6$ 6/group.

A

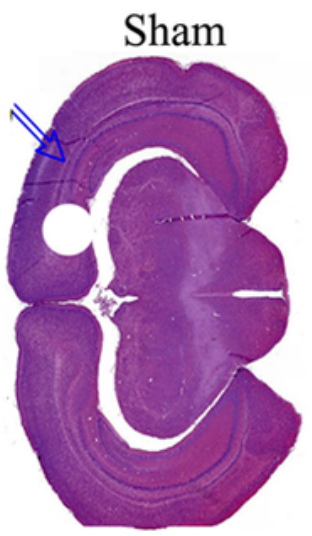

Sham

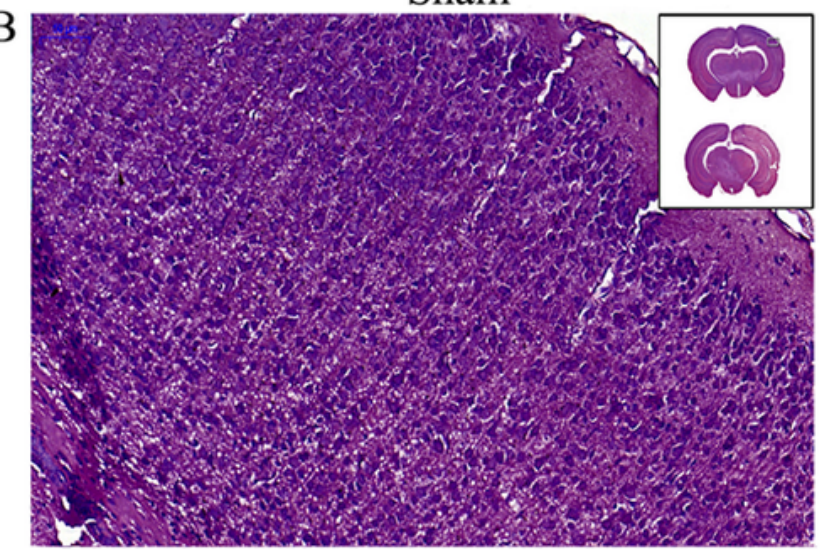

DAPI
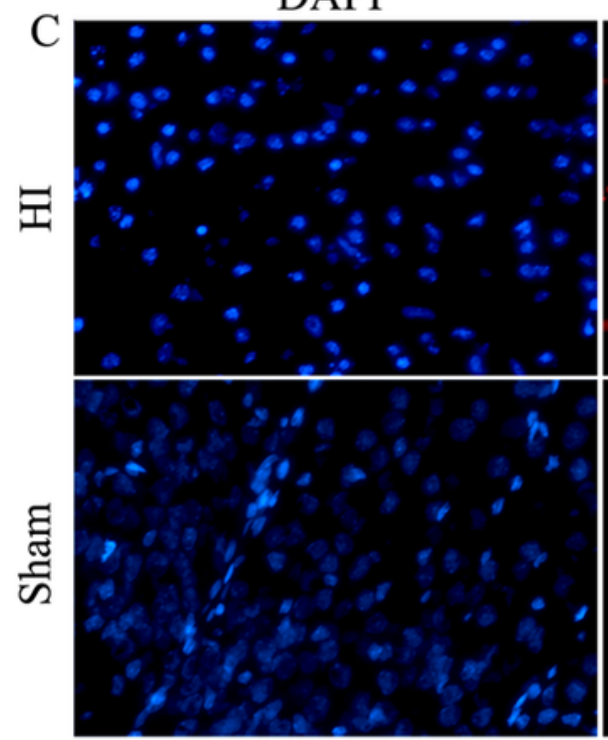

TUNEL
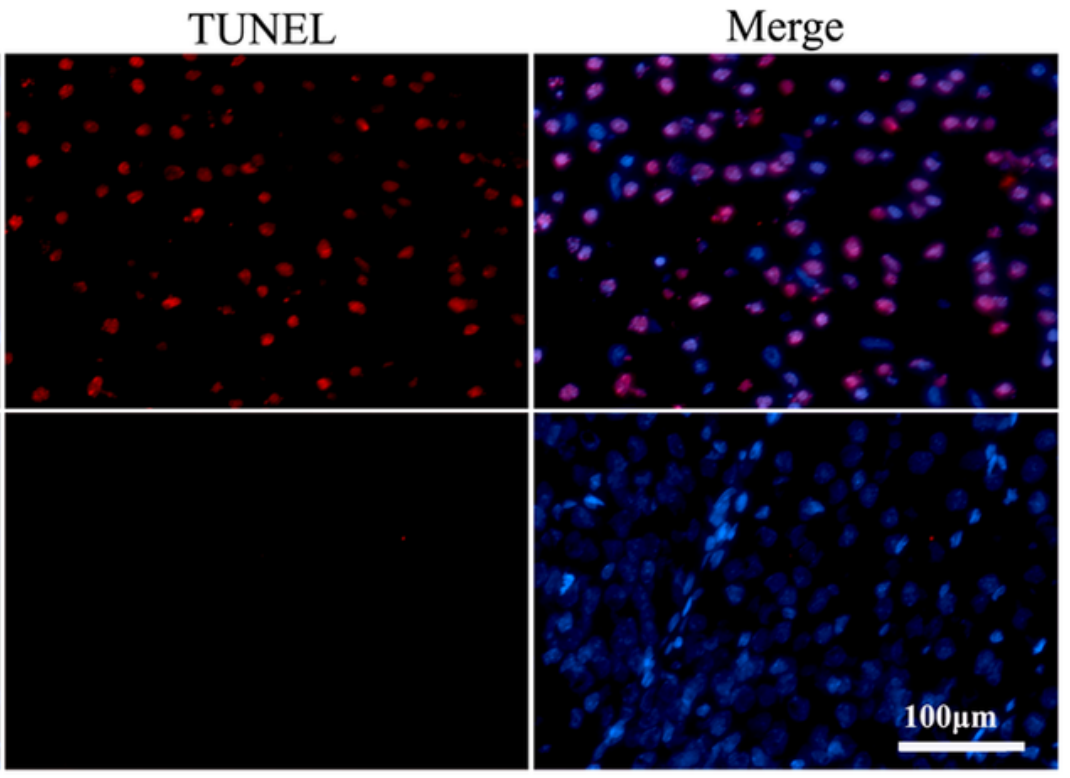


\section{Figure 2}

The effect of $\mathrm{HI}$ on brain tissues (A) The degree of brain edema after HI by HE staining. Scale bar= 200 $\mu \mathrm{m}$. (B) Swelling or necrosis and illegible borders of cell by HE staining. (C) The blue fluorescence stood for DAPI immunostaining, which indicated the location of nucleus, meanwhile, the red fluorescence represented TUNEL immune staining, which indicated the cell apoptosis. HI: hypoxia-ischemia. HE: hematoxylin and eosin. DAPI: 4', 6-diamidino-2-phenylindole. TUNEL: Terminal-deoxynucleoitidyl Transferase Mediated Nick End Labeling. Scale bar $=100 \mu \mathrm{m} . \mathrm{n}=6 /$ group.

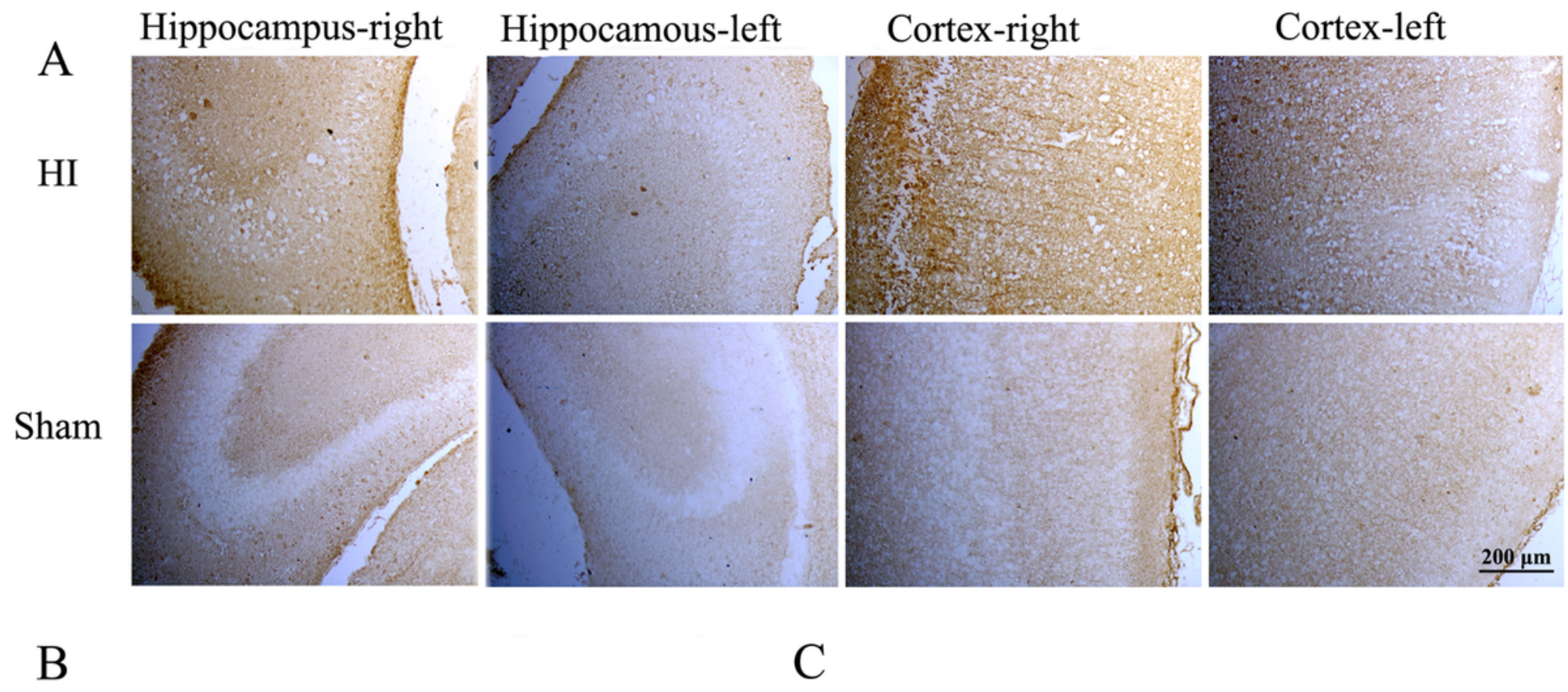

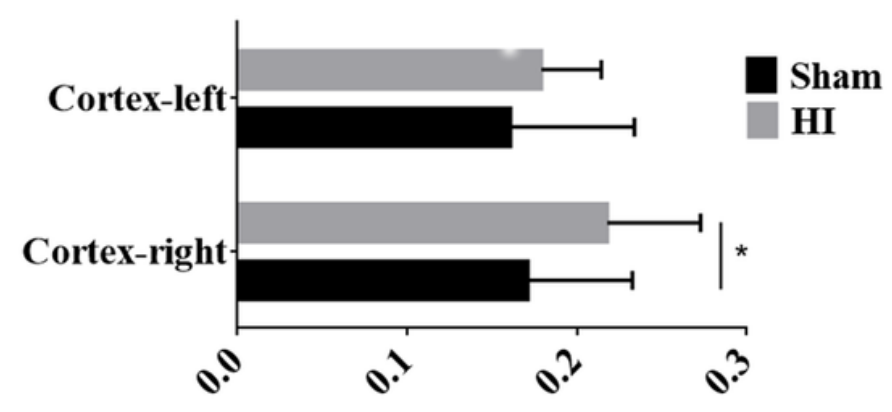

The average optical density of IL-6

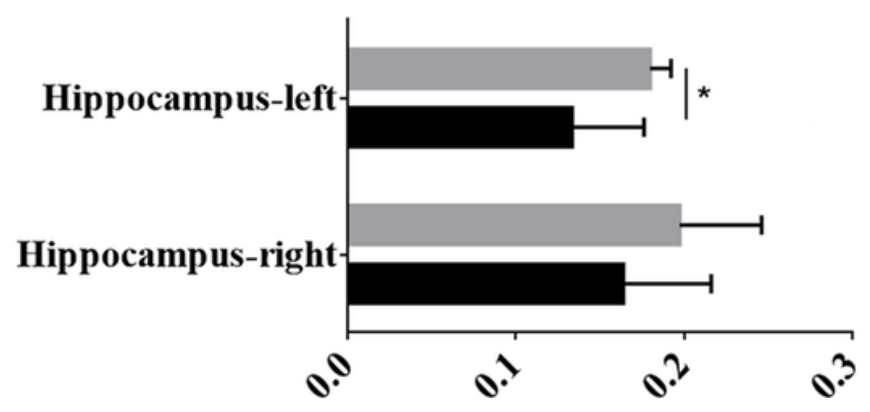

The average optical density of IL-6

\section{Figure 3}

Immunohistochemistry of IL-6 in the Hippocampus and Cortex (A) The location and expression of IL-6 in the hippocampus and cortex in sham and $\mathrm{HI}$ groups by immunohistochemistry. (B, C) Quantitative analysis of the level of IL- 6 in the right and left of hippocampus and cortex in the two groups. HI: hypoxiaischemia. All data are presented as mean \pm SD. ${ }^{*} p<0.05 . n=6 /$ group. Scale bar $=200 \mu \mathrm{m}$. 

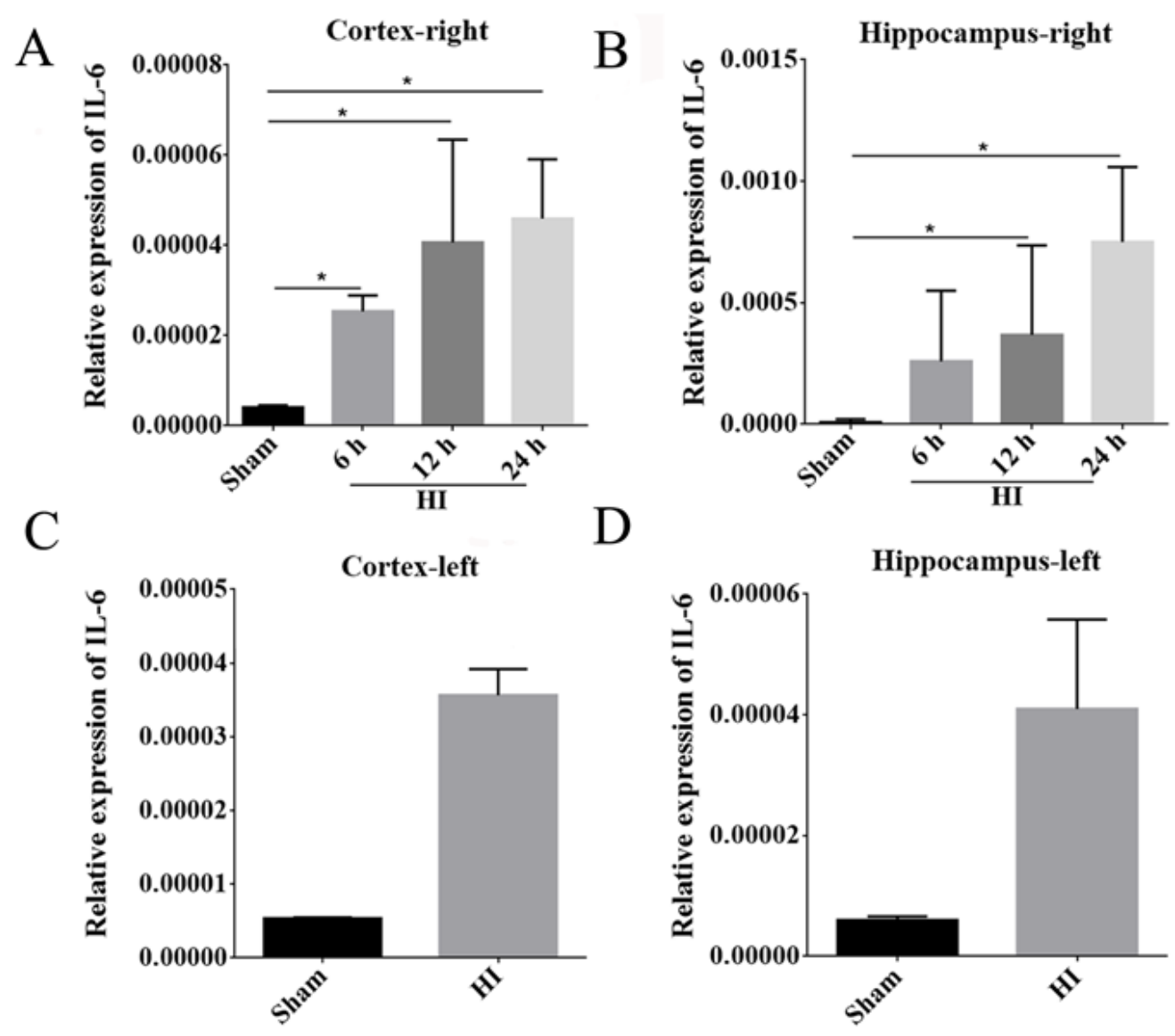

$\mathrm{E}$
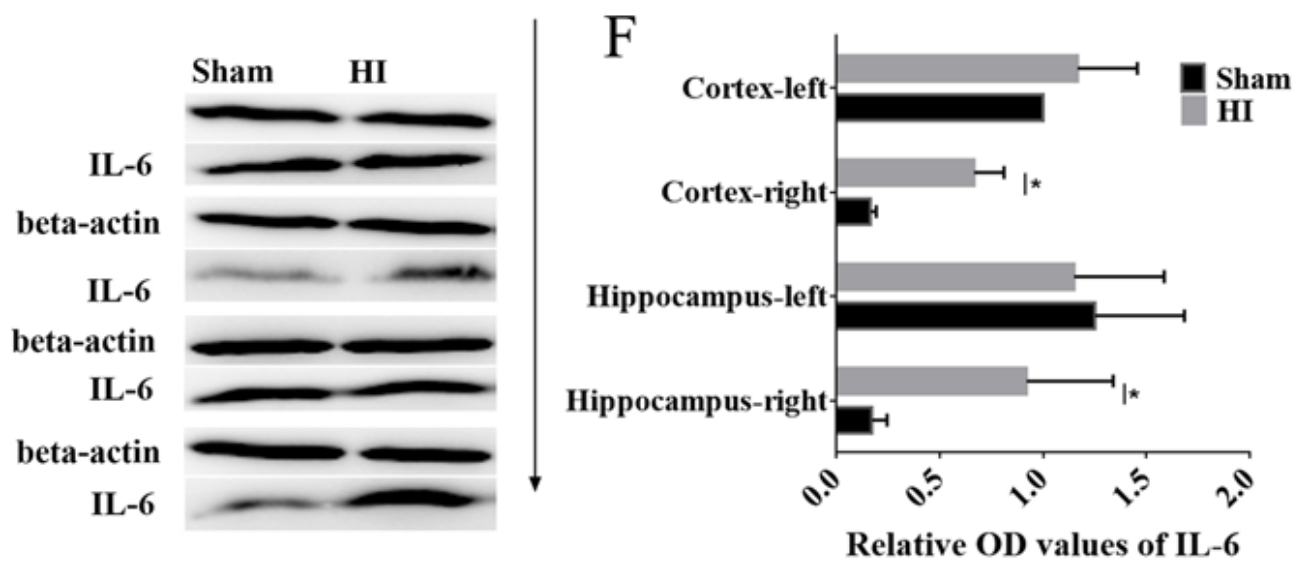

Figure 4

Alteration of IL-6 mRNA expression in the hippocampus and cortex after HI (A-D) The expression of IL-6 mRNA in cortex and hippocampus based on the results of QRT-PCR. ${ }^{*} p<0.05$. (E, F) Western blot showed the expression of IL- 6 protein after $\mathrm{HI}$ in cortex and hippocampus. Beta-actin was treated as an internal control. ${ }^{*} \mathrm{p}<0.05$ : vs. sham group. HI: hypoxia-ischemia. QRT-PCR: quantitative real-time polymerase chain reaction. All data are presented as mean $\pm S D$. $n=6 /$ group. 


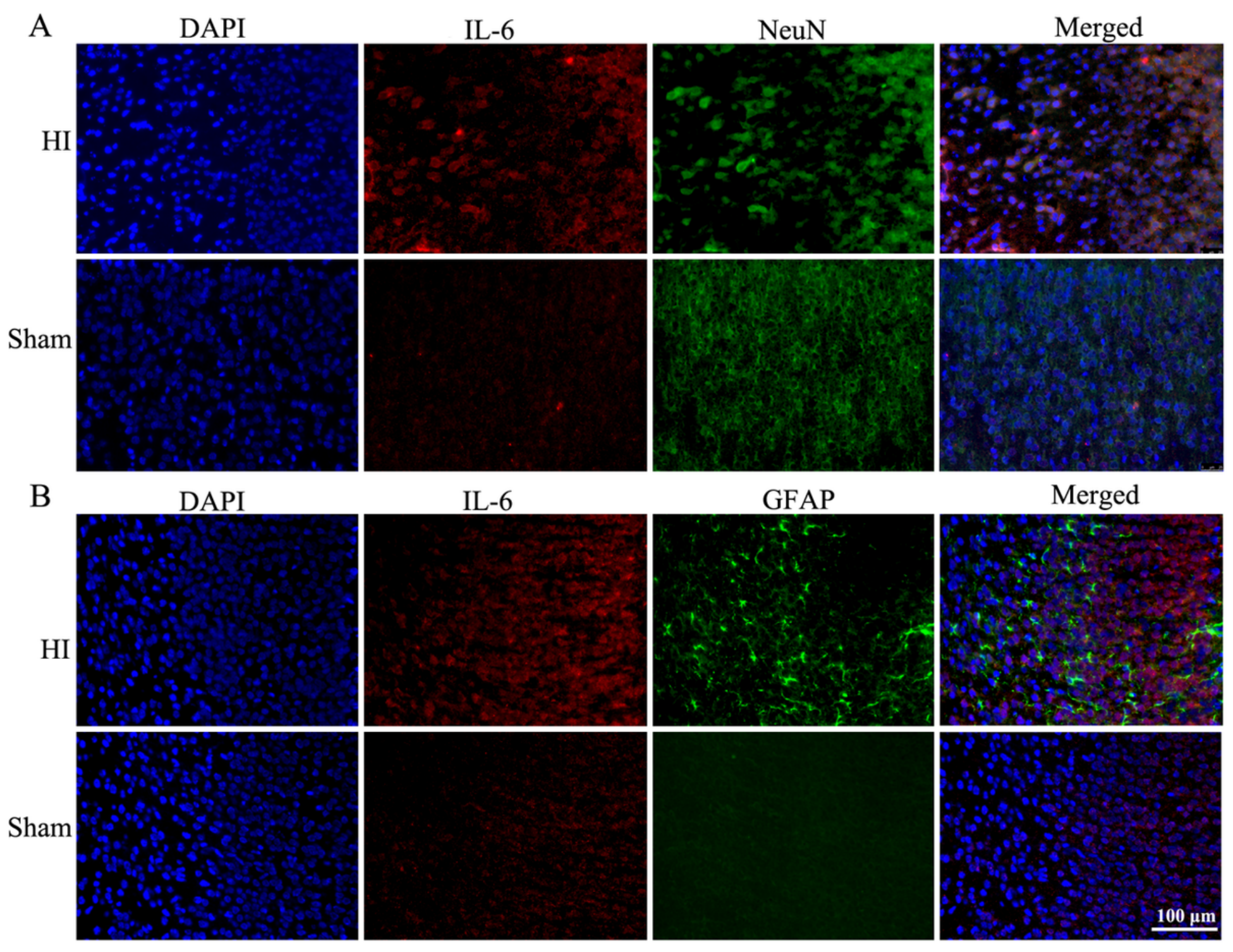

Figure 5

Location of IL- 6 in the neuron and GFAP after HI (A) Immunofluorence staining with IL-6 and NeuN between $\mathrm{HI}$ and sham groups. Blue fluorescence referred to DAPI, which indicated the location of the nucleus, the red fluorescence represented IL-6, the green fluorescence delegated $\mathrm{NeuN}$, which indicated the location of the neuron. (B) Immunofluorence staining of IL-6 and GFAP in the groups of $\mathrm{HI}$ and sham. The nucleuses were stained by blue colors, which are DAPI, IL- 6 was stained by red fluorescence, GFAP was stained by green fluorescence. NeuN: Hexaribonucleotide Binding Protein-3. GFAP: Glial fibrillary acidic protein. DAPI: 4', 6-diamidino-2-phenylindole. Scale bar $=100 \mu \mathrm{m} . \mathrm{n}=6 / \mathrm{group}$. 
A

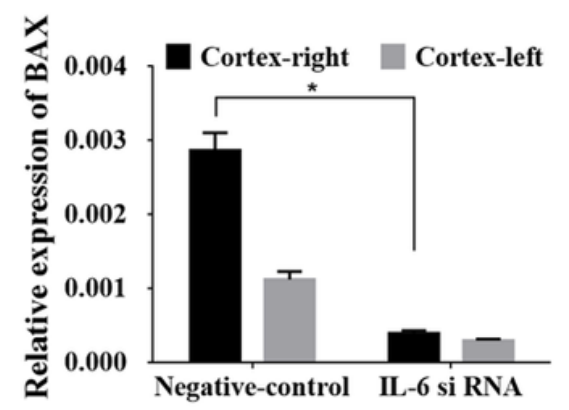

$\mathrm{B}$

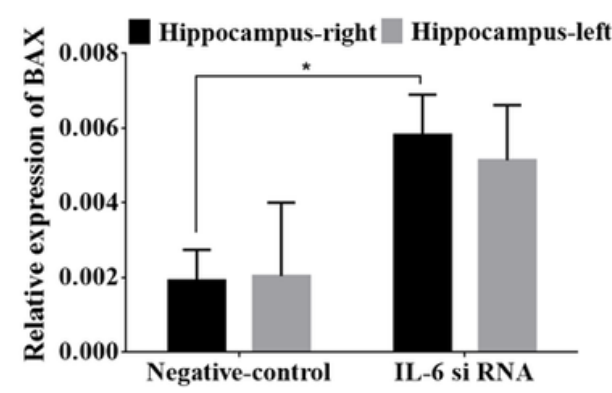

$\mathrm{D}$

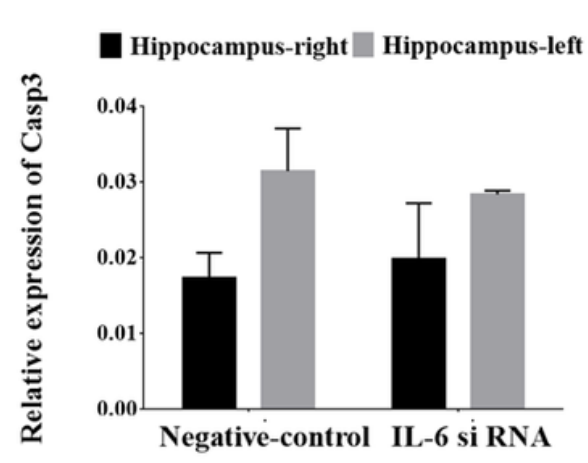

G

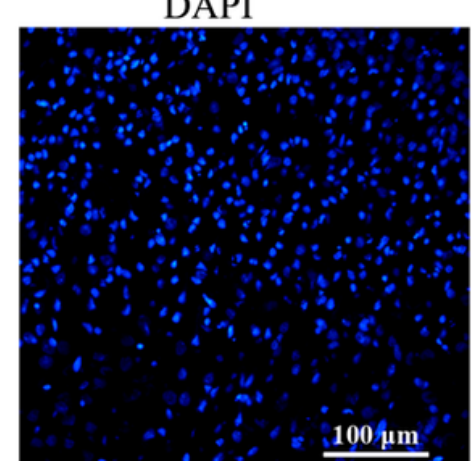

$\mathrm{E}$

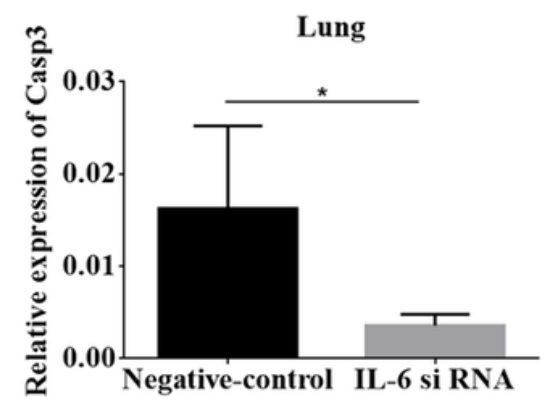

$\mathrm{C}$

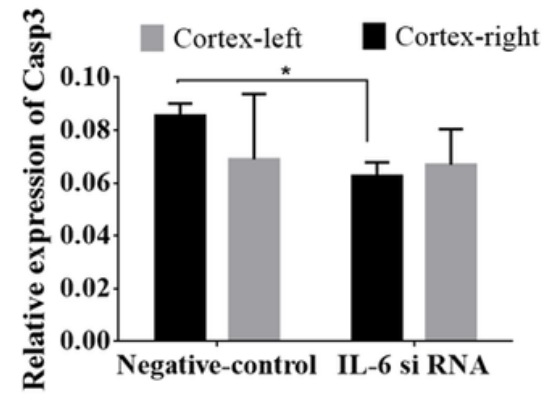

$\mathrm{F}$

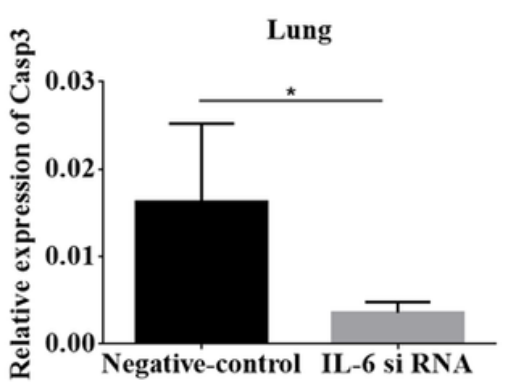

\section{Figure 6}

The relative expression and location of BAX and Casp3 in different organizations after IL- 6 siRNA treatment (A-D) The level of BAX and Casp3 expression in cortex and hippocampus after IL-6 siRNA treatment. $(E, F)$ The histogram showed the expression change of BAX and Casp3 in lung tissues after IL6 siRNA treatment. (G) Double immunofluorescence labeling demonstrated that the colocalization for IL-6 (green) and Casp3 (Red). DAPI: 4', 6-diamidino-2-phenylindole. BAX: Bcl-2-associated X protein. All data are presented as mean \pm SD. ${ }^{*} p<0.05$. Scale bar $=100 \mu \mathrm{m} . \mathrm{n}=6 /$ group. 

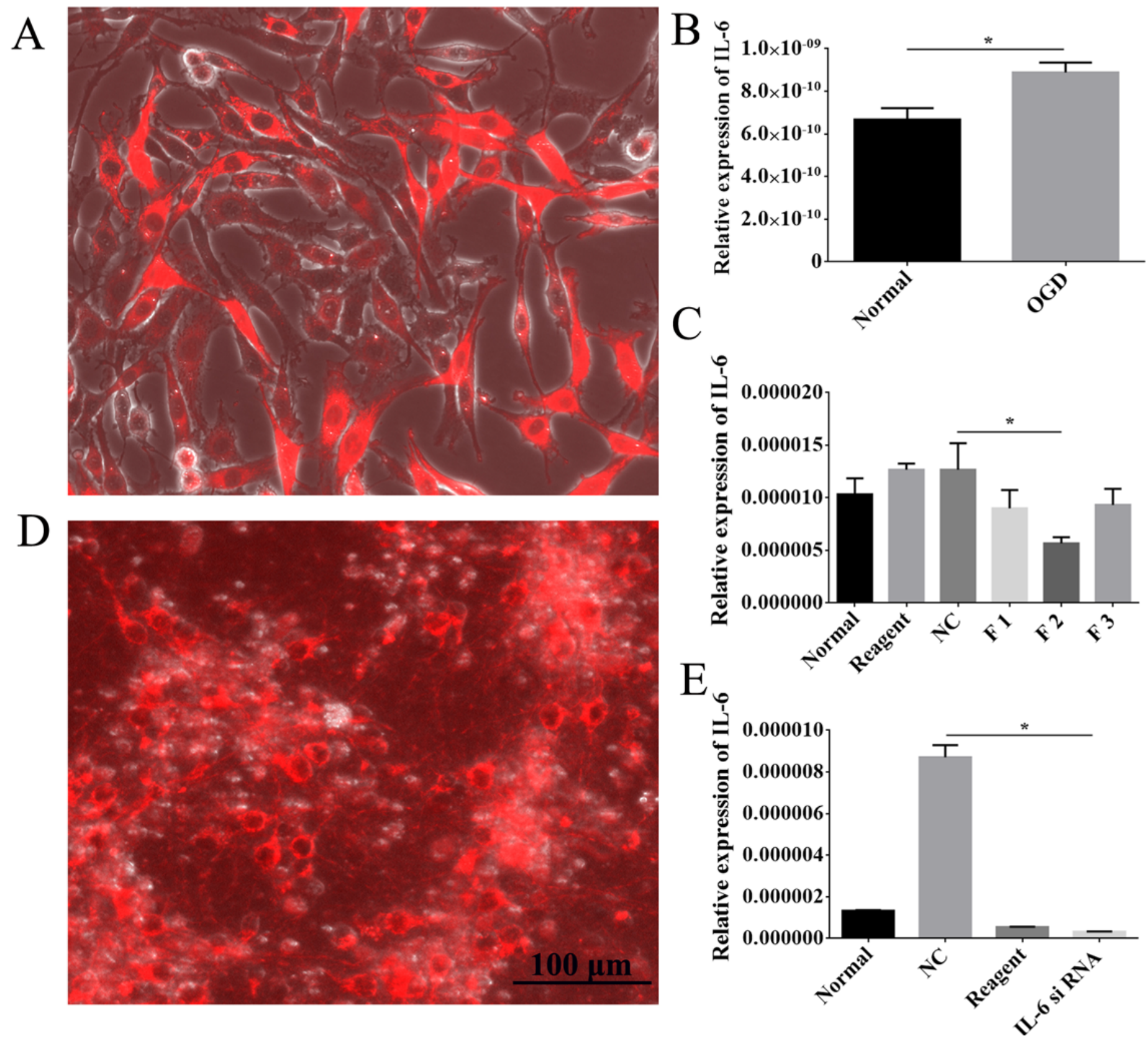

Figure 7

IL-6 RNA interference fragment could downregulate the expression of IL-6 (A) The picture displayed that PC12 cells were successfully transfected with F1, F2 and F3 interference fragments by fluorescent photography. $(B, E)$ The expression of IL-6 after F2 fragment interference into neuron under normal and OGD conditions. (C) Quantitative analysis of the interference efficiency of F1, F2 and F3 fragments. (D) F2 fragment was successfully transfected into neurons. OGD: oxygen-glucose deprivation. siRNA: silencing RNA. F1: treatment with No.1 siRNA fragment, F2: treatment with No.2 siRNA fragment, F3: treatment with No.3 siRNA fragment. All data are presented as mean \pm SD. ${ }^{\star} p<0.05 . n=6 /$ group. Scale bar $=100 \mu \mathrm{m}$. 


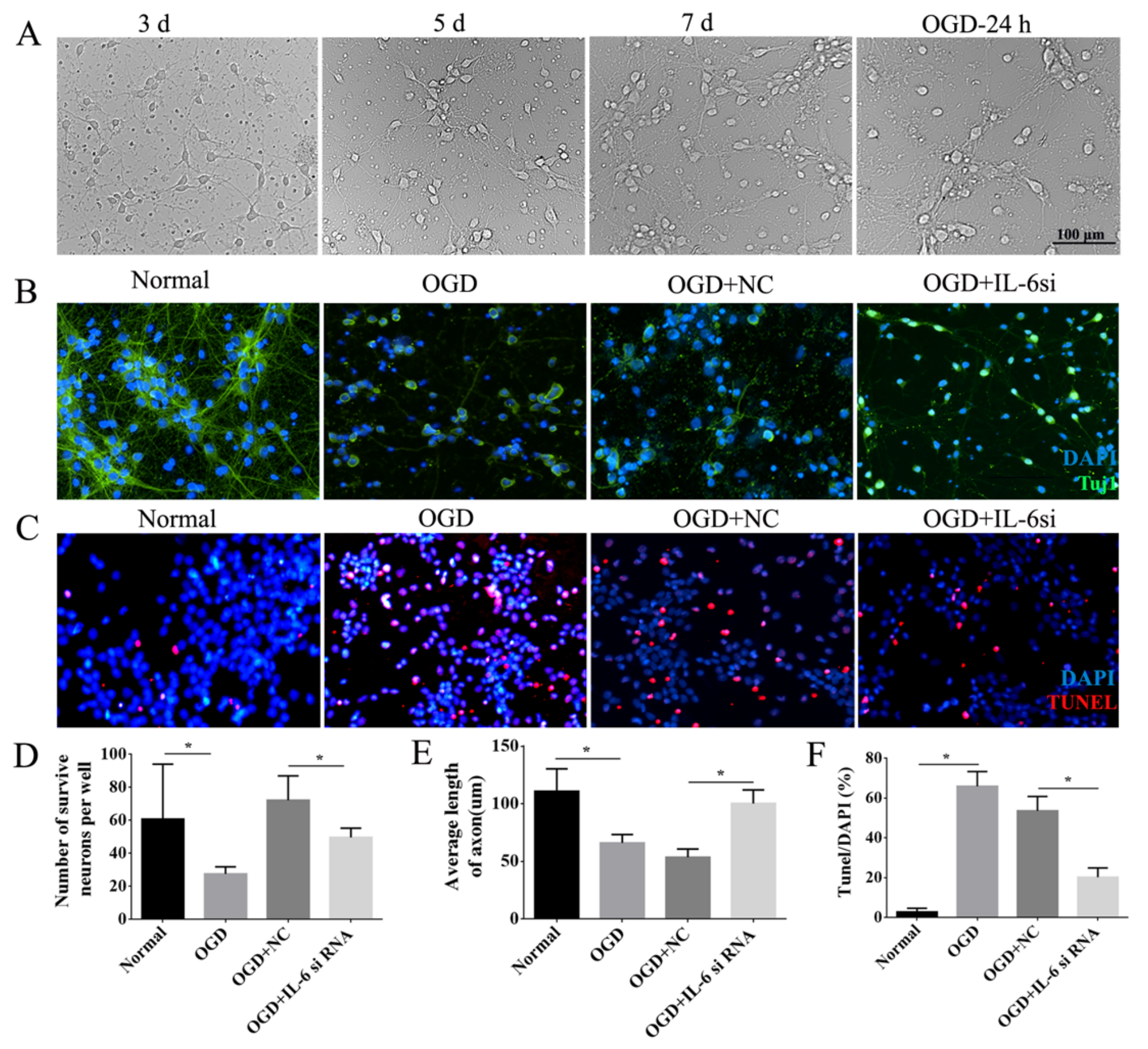

Figure 8

Effect of IL-6 interference on cerebral cortical neurons of OGD. (A) The cortical neurons of bright field picture (200x) in the groups of $3 \mathrm{~d}, 5 \mathrm{~d}, 7 \mathrm{~d}$ in normal neurons and $24 \mathrm{~h}$ in OGD neurons.(B, C) The growth and apoptosis of neurons were observed by immunofluorescence staining of Tuj1 and TUNEL among normal, OGD, OGD+NC and OGD+IL-6si groups. DAPI: nucleus marker (Blue), TUJ1: neuron marker (green), TUNEL: apoptosis marker (red). (D-F) Statistical analysis showed that the number of neurons, average length of axons and apoptosis of neurons after interference with IL-6 under OGD conditions in these groups. OGD: oxygen-glucose deprivation. Tuj1: Neuronal Class III $\beta$-Tubulin. TUNEL: Terminal- 
deoxynucleoitidyl Transferase Mediated Nick End Labeling. NC: negative control. IL-6si: silencing IL-6. All data are presented as mean $\pm S D . * p<0.05$. Scale bar $=100 \mu \mathrm{m} . n=6 /$ group.

A

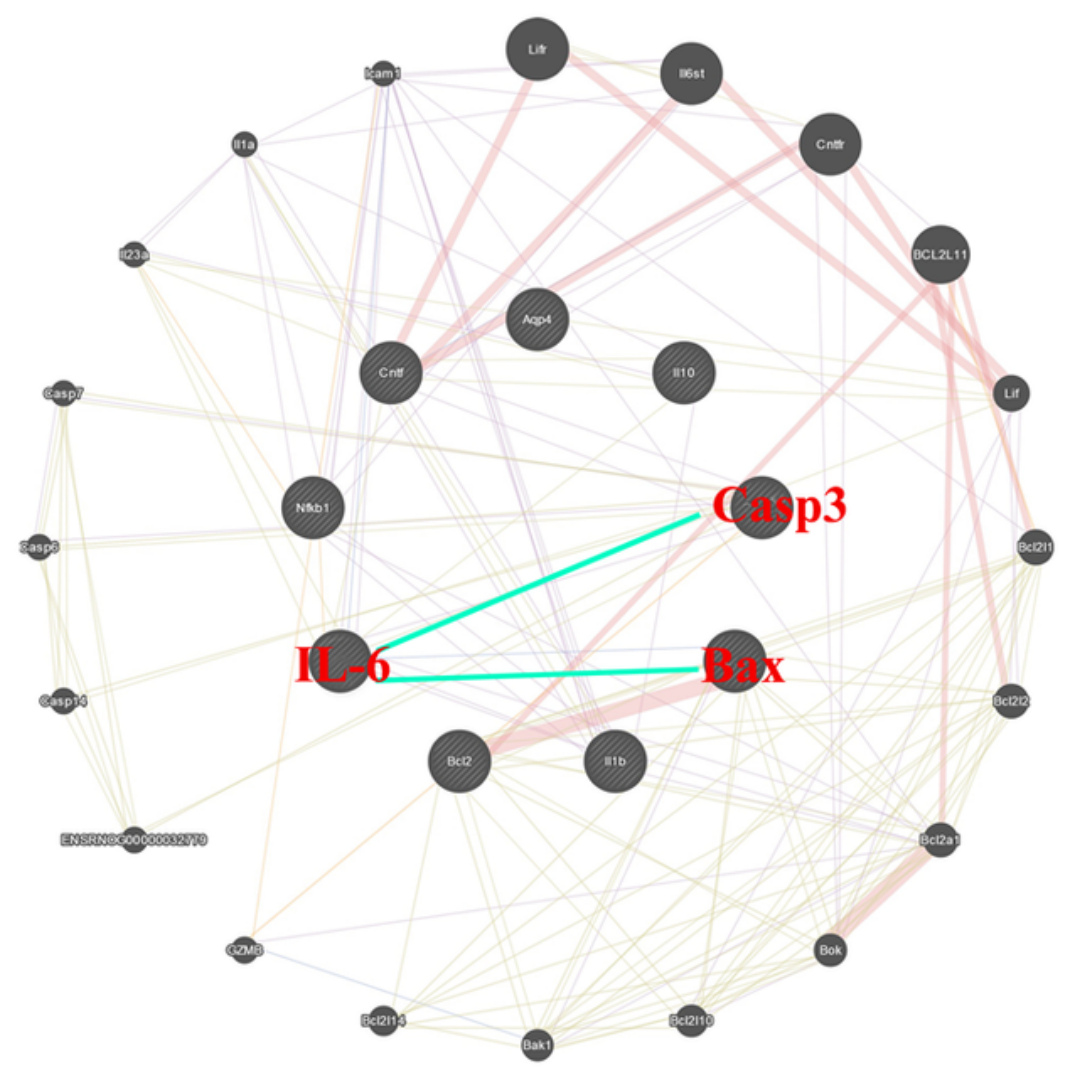

$\mathrm{B}$

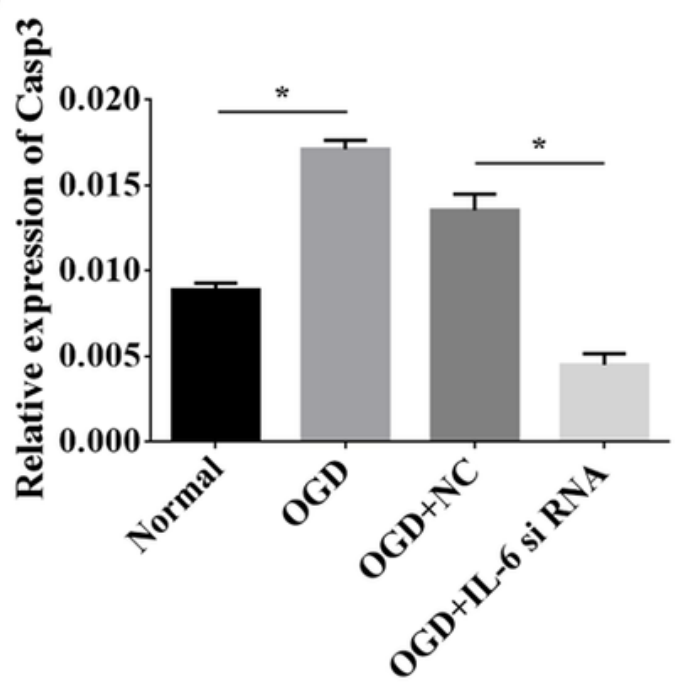

$\mathrm{C}$

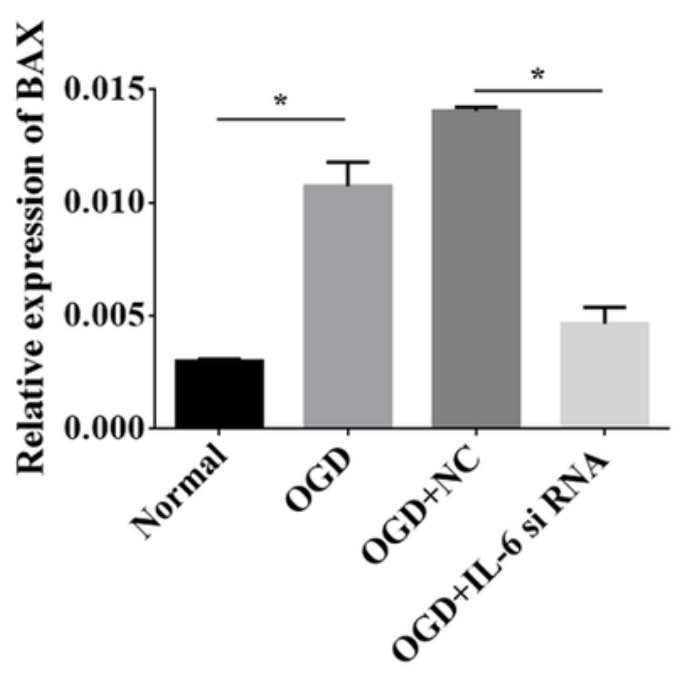

Figure 9

Analysis of IL-6 gene interaction networks (A) The protein-protein interactions network analysis of IL-6, BAX and Casp3. (B, C) Quantitative analysis of the relative expression of BAX and Casp3 in OGD, 
OGD+NC, OGD+IL-6 siRNA groups. OGD: oxygen-glucose deprivation. BAX: Bcl-2-associated X protein. NC: negative control. IL-6si: silencing IL-6. All data are presented as mean \pm SD. ${ }^{\star} p<0.05 . n=6 /$ group.
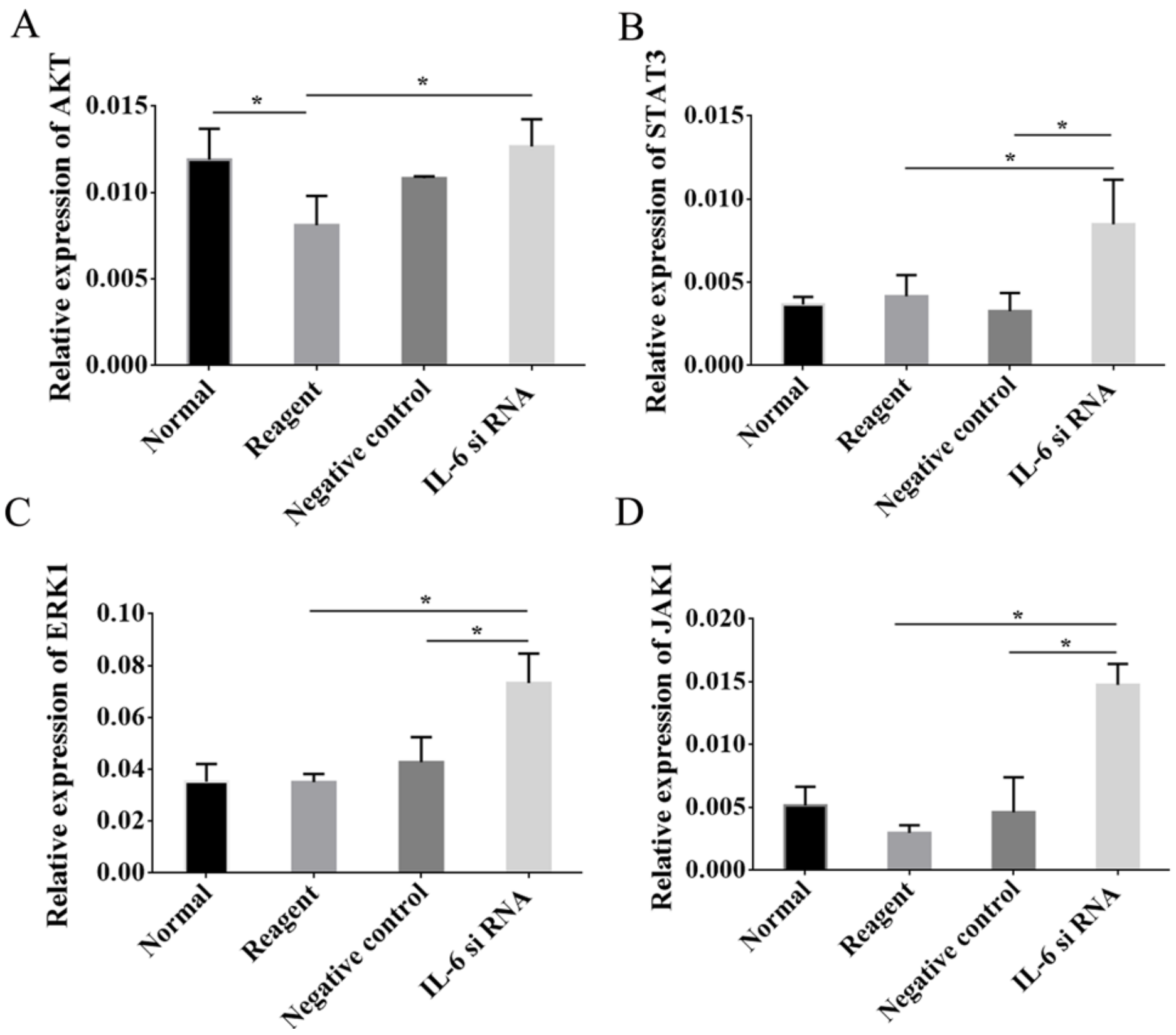

Figure 10

The expression of AKT, STAT3, ERK1 and JAK1 after silencing IL-6 (A-D) The bar chart of relative expression of AKT, STAT3, ERK1, and JAK1 in the groups of normal, reagent, negative control and IL-6 siRNA. AKT: Protein kinase B. STAT3: Signal transducer and activator of transcription 3. ERK1: Mitogenactivated protein kinase 3. JAK1: Janus kinase 1. All data are presented as mean $\pm S D$. ${ }^{*} p<0.05$. $n=$ 6/group. 